\title{
Conversion of bulk kinetic energy into radiation in AGNs and GRBs: Particle transport effects ${ }^{\star}$
}

\author{
R. Vainio ${ }^{1,2}$, M. Pohl ${ }^{3, \star \star}$, and R. Schlickeiser ${ }^{3}$ \\ 1 Space Research Laboratory ${ }^{\star \star \star}$, Department of Physics, 20014 University of Turku, Finland \\ 2 Theoretical Physics Division, Department of Physical Sciences, PO Box 64, 00014 University of Helsinki, Finland \\ 3 Institut für Theoretische Physik, Lehrstuhl IV: Weltraum- und Astrophysik, Ruhr-Universität Bochum, 44780 Bochum, \\ Germany
}

Received 25 May 2002 / Accepted 9 October 2003

\begin{abstract}
We investigate the spatial structure of collisionless collision fronts in relativistic outflows interacting with ambient material. As a result of the interaction, ambient particles are picked up by the outflow and generate transverse plasma waves via streaming instabilities. Pick-up particle transport under the influence of self-generated turbulence inside such interaction regions is studied. We extend our previous momentum space modeling to include also a spatial dimension. We find that the following possibilities are consistent with quasi-linear equations of particle transport and wave generation: (i) if background waves have small intensities inside the outflow region, leading to inefficient scattering across the pitch-angle, $\theta$, of $90^{\circ}$, particles are isotropized in the backward hemisphere (relative to the outflow velocity vector) and self-generated waves have a steep, $\propto k^{-3}$ wavenumber spectrum; (ii) if background waves have large intensities, enabling particles to cross $\theta=90^{\circ}$, particles can be fully isotropized. In case (i), however, the calculated self-generated wave amplitudes are close to the magnitude of the ordered field for reasonable choices of model parameters, giving the particles a chance to be scattered across the resonance gap by non-resonant processes. If the resonance gap is filled, a large fraction of the pick-up particles is expected to return to the upstream region, and an ultra-relativistic shock wave is predicted to form in front of the outflow, where the two relativistic particle populations (ambient and reflected) mix and form a relativistic plasma. Reflection of pick-up protons decreases the $\pi^{0}$-decay luminosity of relativistic outflows, leading to a need to update parameters of previous modeling. An example of outflow parameters reproducing typical TeV-blazar observations is presented.
\end{abstract}

Key words. instabilities - turbulence - galaxies: BL Lacertae Objects: general - gamma rays: theory - shock waves

\section{Introduction}

The observations of sub-hour variability in the high-energy gamma-ray emission from active galactic nuclei (AGNs) (Gaidos et al. 1996), and the discovery that at least a sizable fraction of the enigmatic gamma-ray bursts (GRBs) originate at cosmological distances (Metzger et al. 1997), both place stringent restrictions on the conditions prevalent in the emission regions. In both systems the observations imply a relativistic Doppler amplification of the radiation, for, were that not the case, the gamma-rays would be subject to strong absorption in the emission region due to interactions with

Send offprint requests to: $\mathrm{R}$. Vainio,

e-mail: rami.vainio@helsinki.fi

* Appendices A and B are only available in electronic form at http://www. edpsciences.org

$\star \star$ Now at Department of Physics and Astronomy, Iowa State University, Ames, IA 50011, USA.

$\star \star \star$ Part of Väisälä Institute for Space Physics and Astronomy, University of Turku. ambient photon fields (Pohl 2002). At least for the AGNs the relativistic bulk motion can be directly observed as apparent super-luminal motion of individual emission regions in the socalled jets in sequences of VLBI observations of their radio emission. The Lorentz factors (and Doppler factors) thus derived are of the order of ten for general samples of AGNs (Vermeulen \& Cohen 1994), but may be higher for AGNs showing prominent gamma-ray emission (e.g. Homan et al. 2002, 2003). The range of Lorentz factors prevalent at the time of gamma-ray emission, which presumably occurs before the emission region becomes visible at radio frequencies, is not known, but is likely higher than ten, if the bulk kinetic energy of the jets is the energy reservoir for the particle acceleration.

Though AGNs and GRBs differ in that the latter involves a catastrophic explosion, whereas the former is based on persistent processes fed by black-hole accretion, they clearly share the property that the energetic particles and their radiation products are generated in relativistic outflows, albeit with possibly different degrees of collimation. These recent results have led to a renewed interest in the subject of particle acceleration 
at relativistic collision fronts, for these are an inevitable consequence when relativistic flows encounter ambient material in the interstellar medium of the host galaxy.

Collision fronts are likely sites of particle acceleration by, e.g., Fermi-type processes (Kirk \& Duffy 1999), the time scale of which scales with the ratio of the collision front velocity to the speed of light. Relativistic collision fronts, therefore, make excellent candidates for hosting the very rapid acceleration processes, that are required to explain the fast variability observed from AGNs and GRBs.

In most studies the collision fronts are treated in the framework of ideal MHD, i.e. as a perfect discontinuity with appropriate jump conditions (de Hoffman \& Teller 1950; Ellison \& Reynolds 1991), at which particle acceleration is considered under the test particle assumption (Bednarz \& Ostrowski 1996; Kirk et al. 2000; Achterberg et al. 2001) or accounting for a back-reaction of accelerated particles (Schneider \& Kirk 1987; Pelletier 1999; Ellison \& Double 2002). It is, however, unclear whether the time scale of shock acceleration, which is determined by the nature of the MHD turbulence in the vicinity of the collision front, is short enough to account for the large energies and the rapid variability observed in these objects. Henri et al. (1999) emphasized the role of second-order Fermi acceleration downstream the shock wave noting that Alfvén speeds may be close to the plasma flow speed enabling the stochastic acceleration process to win over the regular one. However, as shown by Vainio \& Schlickeiser $(1998,1999)$ and Vainio et al. (2003), the time scale of the downstream stochastic acceleration by shock-generated turbulence is still longer than that of the regular acceleration, because the downstream wave field is close to unidirectional (at least for quasi-parallel shocks). The relativistic Fermi acceleration (Pelletier 1999) is, on the other hand, much faster when one considers external waves with nonlinear wave amplitudes, e.g., colliding relativistic, Alfvénic solitons that can scatter particles between them.

While the details of particle acceleration at relativistic, hydrodynamical shock fronts have been extensively explored, it is the very assumption of a perfect discontinuity that is questionable. Astrophysical plasmas are collisionless, and thus collision fronts have a finite thickness of the order of the mean free path for the scattering of relativistic particles. The same mean free path determines the probability of multiple crossings of the collision front, so that in general the particles, that are to be accelerated, do not see the collision front as a discontinuity.

A kinetic treatment of relativistic collision fronts is, therefore, desirable. Recently, Pohl \& Schlickeiser (2000) have studied the kinetic relaxation of particles that have traversed a parallel collision front from the upstream to the downstream region. This pick-up process occurs due to scattering off lowwavenumber plasma waves that the pick-up particles generate themselves through streaming instabilities. The calculations, thus, describe the first half-cycle of the standard shock acceleration process for relativistic, collisionless flows. Besides, if the outflow plasma propagating through the jet is dense, the downstream region provides a target for the ultra-relativistic protons to interact with and produce radiation through various leptonic and hadronic emission channels.
The predicted radiation properties resemble those of gamma-ray blazars and, thus, the model offers an attractive alternative to shock acceleration of electrons and subsequent Inverse Compton (IC) scattering. The hadronic $\gamma$-radiation of the pick-up model is a result of the picked-up interstellar protons colliding inelastically with the cold protons of the ejecta. The initial proton energies in these collisions are close to $\Gamma m_{\mathrm{p}} c^{2}$, and the generated pions have typically energies that are a small fraction of this. The $\pi^{0}$-decay photons are the most energetic photons generated in these interactions. Their energies are additionally Doppler boosted by the relativistic, lineof-sight-aligned motion of the downstream medium. The pickup model, nevertheless, requires relatively large values of the outflow Lorentz factors, $\Gamma \gtrsim 100$, to create observable luminosities at $\mathrm{TeV} \gamma$-ray energies. This, however, does not have to contradict the blast-wave Lorentz factors $\Gamma \lesssim 10$ typically deduced from VLBI observations of radio components in AGN jets, because the large values correspond to the initial blast-wave Lorentz factors. While the collimated outflow propagates through the interstellar and/or intergalactic medium, it slows down and can attain a Lorentz factor similar to those observed in the radio jets.

So far, the pick-up process has been studied considering momentum-space behavior of particles, only. Since the downstream region is not loaded with particles uniformly, it is likely that spatial transport of particles is important: after being isotropized by the transverse plasma waves, the particles also start diffusing back toward the collision front. If backscattering by the outflow plasma is significant, a precursor wave could form in front of the outflow. This may significantly change the characteristics of the collision front.

In this paper, we extend the previous modeling efforts to take into account spatial transport of the particles during and after being picked up by transverse waves inside the collimated outflow. In Sect. 2 we will study the spectrum of waves and particle fluxes in a steady state under non-radiative conditions, where the ambient particles are injected at a constant rate into the system and scattered by transverse waves that they amplify/damp themselves, but are not subject to significant energy or catastrophic losses besides escape to the upstream region. The outflow medium is modeled as an electron-proton plasma with magnetic field aligned with the direction of motion, although the results are often directly applicable to a leptonic composition (Schlickeiser et al. 2002), as well. We will consider only pitch-angle scattering by transverse waves and neglect electrostatic instabilities (Pohl et al. 2002). When calculating the spectrum of the waves and the flux of relativistic particles, we will also neglect a possible finite thickness of the outflow medium. In addition to the quasi-linear pitchangle diffusion, we consider a more phenomenological model (relaxation-time approximation) and solve it analytically in the steady state.

Although our study is primarily aimed at a better understanding of the behavior of relativistic collisionless collision fronts, in Sect. 4 we will also briefly discuss to what extent our results have impact on the radiation output in the AGN model of Pohl \& Schlickeiser (2000) (see Fig. 1). For that purpose we will use their notation, where the interstellar number density 


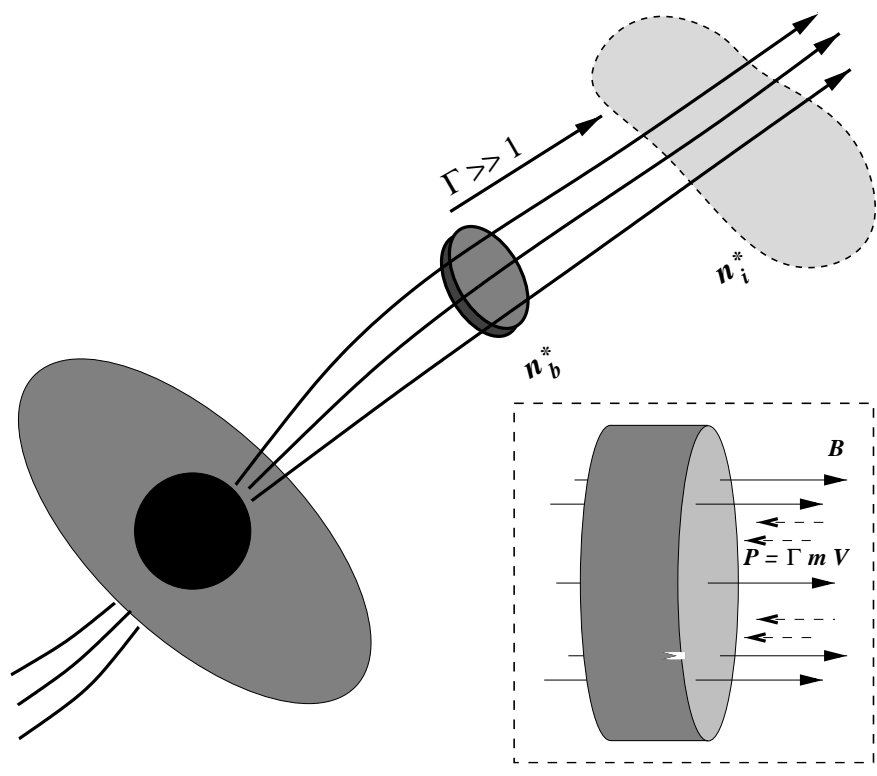

Fig. 1. Collimated outflow model. A cold, dense (density $n_{b}^{*}$ ) plasma cloud ejected from an AGN moves along the magnetic field lines and runs into interstellar/intergalactic gas clouds (density $n_{\mathrm{i}}^{*} \ll n_{b}^{*}$ ) with an ultra-relativistic speed.

is $n_{\mathrm{i}}$, the number density inside the collimated ejecta is $n_{b}$, and the Lorentz-factor of the relative motion of the outflow and the interstellar medium is $\Gamma$. Densities measured in the frame of the interstellar medium are indexed with an asterisk, while those measured in the frame of the outflow are without indexing. The third frame of reference that becomes useful is the frame comoving with the Alfvén waves in the outflow region. As shown by Pohl \& Schlickeiser (2000), the Alfvén waves generated by the streaming instabilities are all backward waves, i.e., propagating anti-parallel (in plasma frame) to the outflow velocity vector. Thus, although the pick-up model involves diffusion of particles in momentum space, this diffusion is in wave-frame pitch angle only and does not lead to stochastic acceleration of particles. Quantities measured in the wave frame are denoted by a tilde. We measure the distance along the axis of the outflow, assumed to coincide with the local magnetic field direction, by $x$. This axis is pointing nearly toward the observer in the interstellar medium, and positive $x$-component of velocity, thus, means particles moving parallel to the outflow motion.

\section{The spatial transport of pick-up particles}

\subsection{Quasi-linear model}

We assume that the system has attained a steady state, where the intensity of the waves and the particle distributions are constant over time, but vary as a function of position. We further assume that the waves in the system are (as in the homogeneous case) all propagating in the backward direction in the steady state. Using a mixed coordinate system, where position $x$ (and time) are measured in the blob frame but the particle momentum $\tilde{p}$ and pitch-angle cosine $\tilde{\mu}$ in the Alfvén wave frame (denoted by tilde $\sim$ ), we can write the particle distribution function as

$f_{j}(x, \tilde{p}, \tilde{\mu})=F_{j}(x, \tilde{\mu}) \frac{\delta\left(\tilde{p}-m_{j} \tilde{\Gamma} \tilde{V}\right)}{2 \pi \tilde{p}^{2}}$.

Although $f_{j}$ is treated as a function of mixed coordinates, it is still the invariant distribution function, where all the components of space-time and momentum are measured in the same frame of reference, i.e., it is not equal to $\mathrm{d}^{6} N /\left(\mathrm{d}^{3} x \mathrm{~d}^{3} \tilde{p}\right)$. Thus, integrating $f_{j}$ over $\tilde{p}$ space gives the particle density measured in the wave frame.

The evolution of the particle density over $\tilde{\mu}$,

$F_{j}(x, \tilde{\mu})=2 \pi \int \mathrm{d} \tilde{p} \tilde{p}^{2} f_{j}(x, \tilde{p}, \tilde{\mu})$

is governed by (Kirk et al. 1988)

$\Gamma_{\mathrm{A}}\left(\tilde{V} \tilde{\mu}-V_{\mathrm{A}}\right) \frac{\partial F_{j}}{\partial x}=\frac{\partial}{\partial \tilde{\mu}} \tilde{D}_{\mu \mu}^{(j)} \frac{\partial F_{j}}{\partial \tilde{\mu}}$

where $V_{\mathrm{A}}$ is the Alfvén speed inside the ejecta, $\Gamma_{\mathrm{A}}=(1-$ $\left.V_{\mathrm{A}}^{2} / c^{2}\right)^{-1 / 2}$, and

$$
\begin{aligned}
\tilde{D}_{\mu \mu}^{(j)}(\tilde{\mu}, x)= & \pi \frac{\Omega_{j}^{2}}{2 B^{2} \tilde{\Gamma}^{2}}\left(1-\tilde{\mu}^{2}\right) \\
& \times \int_{-\infty}^{+\infty} \mathrm{d} \tilde{k} \tilde{I}(\tilde{k}, x) \delta\left(\tilde{k} \tilde{V} \tilde{\mu}+\frac{\Omega_{j}}{\tilde{\Gamma}}\right) \\
= & \pi \frac{\Omega_{j}^{2}}{2 \tilde{V}\left|\tilde{\mu}^{2}\right| \tilde{\Gamma}^{2} B^{2}}\left(1-\tilde{\mu}^{2}\right) I\left(k_{j}, x\right)
\end{aligned}
$$

is the pitch-angle diffusion coefficient due to Alfvén waves. Here, $\tilde{I}(\tilde{k}, x)$ is the wave-frame intensity of the Alfvén waves evaluated at the resonant (wave-frame) wavenumber

$k_{j}(\tilde{\mu})=-\frac{\Omega_{j}}{\tilde{\Gamma} \tilde{V} \tilde{\mu}}$.

The wave evolution is governed by the equation (see Appendix A)

$-V_{\mathrm{A}} \frac{\partial \tilde{I}}{\partial x}=\sigma \tilde{I}$

$=-\frac{4 \pi}{\Gamma_{\mathrm{A}}} \sum_{j=e, p} H\left(|\tilde{k}|-\tilde{R}_{j}^{-1}\right) \frac{m_{j}\left|\Omega_{j}\right| V_{\mathrm{A}}}{\tilde{k}^{2}}\left(\tilde{D}_{\mu \mu}^{(j)} \frac{\partial F_{j}}{\partial \tilde{\mu}}\right)_{\tilde{\mu}=\mu_{j}}$

with

$\mu_{j}(\tilde{k})=-\frac{\Omega_{j}}{\tilde{\Gamma} \tilde{V} \tilde{k}} \quad$ and $\quad \tilde{R}_{j}=\frac{\tilde{\Gamma} \tilde{V}}{\left|\Omega_{j}\right|}$,

so that $\tilde{R}_{j}$ is the particle's wave-frame Larmor radius.

We can integrate the particle transport equation once over $\tilde{\mu}$ from -1 to $\mu_{j}$ to get

$\Gamma_{\mathrm{A}} \int_{-1}^{\mu_{j}} \mathrm{~d} \tilde{\mu}\left(\tilde{V} \tilde{\mu}-V_{\mathrm{A}}\right) \frac{\partial F_{j}}{\partial x}=\left(\tilde{D}_{\mu \mu}^{(j)} \frac{\partial F_{j}}{\partial \tilde{\mu}}\right)_{\tilde{\mu}=\mu_{j}}$,

so on using Eq. (7)

$$
\begin{aligned}
\frac{\partial \tilde{I}}{\partial x}= & 4 \pi \sum_{j=e, p} H\left(|\tilde{k}|-\tilde{R}_{j}^{-1}\right) \frac{m_{j}\left|\Omega_{j}\right|}{\tilde{k}^{2}} \\
& \times \int_{-1}^{\mu_{j}} \mathrm{~d} \tilde{\mu}\left(\tilde{V} \tilde{\mu}-V_{\mathrm{A}}\right) \frac{\partial F_{j}}{\partial x} .
\end{aligned}
$$


This can be integrated over position to yield

$$
\begin{aligned}
& \tilde{I}(\tilde{k}, x)-\tilde{I}(\tilde{k}, 0)=4 \pi \sum_{j=e, p} H\left(|\tilde{k}|-\tilde{R}_{j}^{-1}\right) \frac{m_{j}\left|\Omega_{j}\right|}{\tilde{k}^{2}} \\
& \times \int_{-1}^{\mu_{j}} \mathrm{~d} \tilde{\mu}\left(\tilde{V} \tilde{\mu}-V_{\mathrm{A}}\right)\left[F_{j}(x, \tilde{\mu})-F_{j}(0, \tilde{\mu})\right]
\end{aligned}
$$

We know as boundary conditions the values of $\tilde{I}(\tilde{k}, 0)=I_{0}(\tilde{k})$ and $F_{j}\left(0, \tilde{\mu} \leq V_{\mathrm{A}} / \tilde{V}\right)=\tilde{n}_{\mathrm{i}} \delta(\tilde{\mu}+1)$ with $\tilde{n}_{\mathrm{i}}=n_{\mathrm{i}} /\left[\Gamma_{\mathrm{A}}(1+\right.$ $\left.\left.\tilde{V} V_{\mathrm{A}} / c^{2}\right)\right]$ being the ambient particle density as measured in the wave frame. We do not know $F_{j}\left(0, \tilde{\mu}>V_{\mathrm{A}} / \tilde{V}\right)$; this would have to be calculated. We also know that the $x$ and $\tilde{\mu}$ derivatives vanish as $x \rightarrow-\infty$. We do not, however, know the value of the particle density at infinity, so this equation, in contrast to its counterpart in the homogeneous time-dependent case, does not fully specify the spectrum of waves in the final state.

We know from Eq. (8) that particle streaming (in the blob frame) is constant in the steady state, i.e.,

$\int_{-1}^{+1} \mathrm{~d} \tilde{\mu}\left[\left(\tilde{V} \tilde{\mu}-V_{\mathrm{A}}\right) F_{j}(x, \tilde{\mu})-\left(\tilde{V} \tilde{\mu}-V_{\mathrm{A}}\right) F_{j}(0, \tilde{\mu})\right]=0$.

Assuming that scattering produces an isotropic particle distribution at large distances from the collision front, we may write the final particle streaming, using a transmission coefficient, $T_{j}$, in the form

$-2 V_{\mathrm{A}} F_{j}(-\infty, \tilde{\mu})=-\left(\tilde{V}+V_{\mathrm{A}}\right) \tilde{n}_{\mathrm{i}} T_{j}$

where

$$
\begin{aligned}
1-T_{j} & \equiv-\frac{\int_{V_{\mathrm{A}} / \tilde{V}}^{1} \mathrm{~d} \tilde{\mu}\left(\tilde{V} \tilde{\mu}-V_{\mathrm{A}}\right) F_{j}(0, \tilde{\mu})}{\int_{-1}^{V_{\mathrm{A}} / \tilde{V}} \mathrm{~d} \tilde{\mu}\left(\tilde{V} \tilde{\mu}-V_{\mathrm{A}}\right) F_{j}(0, \tilde{\mu})} \\
& =\frac{\int_{V_{\mathrm{A}} / \tilde{V}}^{1} \mathrm{~d} \tilde{\mu}\left(\tilde{V} \tilde{\mu}-V_{\mathrm{A}}\right) F_{j}(0, \tilde{\mu})}{\left(\tilde{V}+V_{\mathrm{A}}\right) \tilde{n}_{\mathrm{i}}}>0
\end{aligned}
$$

gives the reflection coefficient, i.e., the fraction of $j$-particle flux that gets backscattered to the upstream region. Note that $1-T_{j}<1$. Thus, at wavenumbers resonant with particles at $\tilde{\mu}<V_{\mathrm{A}} / \tilde{V}$, we can use Eq. (10) to write

$$
\begin{gathered}
\tilde{I}(\tilde{k},-\infty)-\tilde{I}_{0}(\tilde{k})=4 \pi\left(\tilde{V}+V_{\mathrm{A}}\right) \tilde{n}_{\mathrm{i}} \sum_{j=e, p} H\left(|\tilde{k}|-\tilde{R}_{j}^{-1}\right) \\
\times \frac{m_{j}\left|\Omega_{j}\right|}{\tilde{k}^{2}}\left[1-T_{j} \frac{1+\mu_{j}(\tilde{k})}{2 V_{\mathrm{A}}}\left(\tilde{V} \frac{1-\mu_{j}(\tilde{k})}{2}+V_{\mathrm{A}}\right)\right] .
\end{gathered}
$$

The term in brackets is positive for all $\mu_{j}$ as long as

$$
T_{j} \leq \frac{4 \tilde{V} V_{\mathrm{A}}}{\left(\tilde{V}+V_{\mathrm{A}}\right)^{2}}=1-\left(\frac{\tilde{V}-V_{\mathrm{A}}}{\tilde{V}+V_{\mathrm{A}}}\right)^{2},
$$

where we identify the last term as the ratio of forward to backward flux for an isotropic (in the wave frame) particle distribution. This is the exact value of the reflection coefficient for such a particle distribution. It may, thus, be regarded as the upper limit of the reflection coefficient in our case, since it is unphysical to assume that a beamed distribution of incoming particles would result in more reflected particles than an isotropic one.

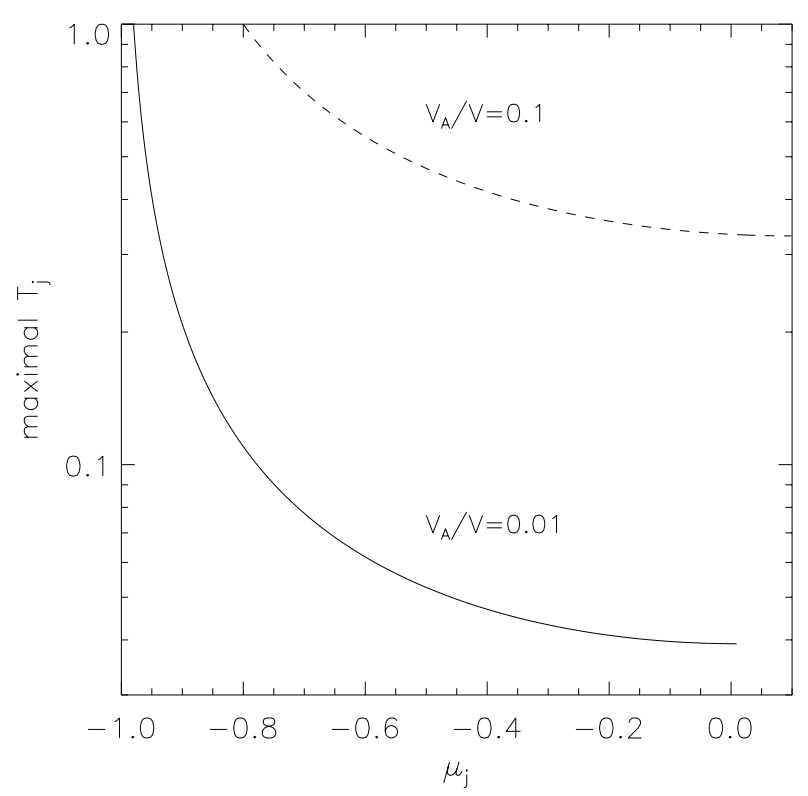

Fig. 2. Maximal transmission coefficient as a function of resonant pitch-angle cosine at $\mu_{j} \leq V_{\mathrm{A}} / \tilde{V}$.

We have plotted, in Fig. 2, the maximum value of $T_{j}$ as a function of $\mu_{j}$, i.e., requiring that the self-generated wave spectrum at $\tilde{\mu}=\mu_{j}$ is zero. Thus, the self-generated spectrum is positive for $\tilde{\mu}<\mu_{j}\left(T_{j}\right)$, and negative for $\mu_{j}\left(T_{j}\right)<\tilde{\mu}<V_{\mathrm{A}} / \tilde{V}$ for $T_{j}$ given in Fig. 2. (A negative self-generated spectrum is reasonable only if there exist enough background waves to make the total spectrum positive everywhere.) The minimum value of the curve is always obtained at $\mu_{j}=V_{\mathrm{A}} / \tilde{V}$. To obtain a solution with a fully positive self-generated part, one would, thus, be forced to choose

$T_{j}=\frac{4 \tilde{V} V_{\mathrm{A}}}{\left(\tilde{V}+V_{\mathrm{A}}\right)^{2}}$.

Other possibilities are discussed in detail in Appendix B. The main conclusions are that under the quasi-linear approximation, the transmission coefficient can not be much larger than the value of Eq. (16) and that the quasi-linear solutions necessarily become dependent on the details of particle scattering across the regions of small $\tilde{\mu}$. For any physically allowed value of the transmission coefficient, it seems, our calculation yields a particle mean free path dependent on the spectrum of background and/or forward waves, neither of which can be fixed by the present calculation.

One type of solution allowed by the quasi-linear equations still needs to be discussed. At least in principle, a solution with no scattering across $\tilde{\mu}=0$ is allowed, because the form of the self-generated spectrum yields an infinite mean free path: $\tilde{I}(\tilde{k})-$ $\tilde{I}_{0}(\tilde{k}) \propto \tilde{k}^{-2}$ asymptotically even if electron-generated waves can scatter protons across $\tilde{\mu}=V_{\mathrm{A}} / \tilde{V}$ and vice versa. In this case, all particles are transmitted to the downstream region and the final particle distribution will be isotropic in the backward hemisphere. Since the streaming in the backward hemisphere is conserved, we get,

$-\frac{\tilde{V}+2 V_{\mathrm{A}}}{2} F_{j}(-\infty, \tilde{\mu})=-\left(\tilde{V}+V_{\mathrm{A}}\right) \tilde{n}_{\mathrm{i}}$ 
In this case, the wave spectrum would be

$$
\begin{gathered}
\tilde{I}(\tilde{k},-\infty)=I_{0}(\tilde{k})+4 \pi \sum_{j=e, p} H\left(|\tilde{k}|-\tilde{R}_{j}^{-1}\right) H\left(q_{j} \tilde{k}\right) \\
\times \frac{\tilde{\Gamma} m_{j} \tilde{V}\left(\tilde{V}+V_{\mathrm{A}}\right) \tilde{n}_{\mathrm{i}}}{\tilde{k}}\left(\frac{\Omega_{j}}{\tilde{\Gamma} \tilde{V} \tilde{k}}\right)^{2} \frac{\Omega_{j} / \tilde{\Gamma} \tilde{k}+2 V_{\mathrm{A}}}{\tilde{V}+2 V_{\mathrm{A}}},
\end{gathered}
$$

which has an asymptotic $1 / \tilde{k}^{3}$ behavior, consistent with the infinite mean free path (related to the inhibited crossing of $\tilde{\mu}=0$ ). This means that for a steep background spectrum of backward waves only, particles are not isotropized via the transverse instability. Note also, that this particle distribution damps forward waves, consistent with the assumption of an infinite mean free path. Estimating the amplitude of the self-generated waves from this solution, however, yields a very large value, $\delta \tilde{B} \sim B$, implying that particles may find other, non-resonant ways to scatter across $\tilde{\mu}=0$, even effectively. We will, next, consider a simplified scattering model to explore this possibility.

\subsection{Relaxation-time model}

Including particle transport effects to the quasi-linear model seems to lead to difficulties in predicting the exact value of the particle flux and the angular distribution at $x \rightarrow \infty$, which is crucial for the radiation output. If there exists a handsome amount of scattering across $\mu=0$, like in a model including some kind of resonance broadening (see, e.g., Schlickeiser \& Achatz 1993; Vainio \& Laitinen 2001, for a few possibilities), there is no simple way to calculate the value of the transmission coefficient exactly. We can, however, obtain an estimate for a simplified scattering operator, i.e., using the relaxationtime approximation.

The relaxation-time approximation is often used to model scattering in large amplitude turbulence, like turbulence around shock waves (Ellison et al. 1996). The application of the model lends support from the finding above that the numerical estimates yield a self-generated turbulent magnetic field that has an amplitude approaching the mean magnetic field in the downstream plasma. This may also be the case with the background waves coming in from the upstream medium. This can occur, since the large Lorentz factor of the outflow boosts the transverse ambient fields to very large values. Transverse electric field and wave frequency are conserved at the boundary. Using Faraday's law gives a relation $\delta B / k \sim \delta B_{i} / k_{i}$ for the field amplitude downstream, $\delta B$, to that upstream, $\delta B_{i}$. Here, $V_{\mathrm{A}} k \sim c k_{i}$ and $\delta B_{i} \sim \Gamma^{2} \delta B_{i}^{*}$, if the wave electric fields and frequencies can be neglected in the ambient medium in its rest frame, i.e., if $\Gamma_{\mathrm{A}} \approx 1$ in the ambient interstellar medium. For the large considered density contrasts, $n_{b} / n_{\mathrm{i}}^{*}$, this is not the case, however taking the wave electric fields and frequencies into account would further increase the amplitude of incoming waves. Thus, typically used values give an estimate of $\left(\delta B_{0}\right)^{2} \sim 10^{8}\left(\delta B_{i}^{*}\right)^{2}$, which can clearly be large compared to the mean magnetic field.
Let us study the following kinetic equation for particles in the downstream plasma $(x \leq 0)$ that have experienced at least one isotropizing scattering

$\Gamma_{\mathrm{A}}\left(\tilde{V} \tilde{\mu}-V_{\mathrm{A}}\right) \frac{\partial F}{\partial x}=\frac{\tilde{n} / 2-F}{\tau(x)}+\frac{Q(x)}{2}$,

where

$\tilde{n}(x)=\int_{-1}^{+1} \mathrm{~d} \tilde{\mu} F(\tilde{\mu}, x)$

is the particle density, and

$Q(x)=\frac{\tilde{n}_{\mathrm{i}}}{\tau(x)} \exp \left\{\int_{0}^{x} \frac{\mathrm{d} x^{\prime}}{\Gamma_{\mathrm{A}}\left(\tilde{V}+V_{\mathrm{A}}\right) \tau\left(x^{\prime}\right)}\right\}$

is the source function related to the relaxation (with the time constant $\tau$ ) of the incoming beam of ambient particles. We, first, integrate the kinetic equation over $\tilde{\mu}$ to get

$\Gamma_{\mathrm{A}} \frac{\partial}{\partial x}\left[\tilde{S}(x)-V_{\mathrm{A}} \tilde{n}(x)\right]=Q(x)$

where

$\tilde{S}(x)=\tilde{V} \int_{-1}^{+1} \mathrm{~d} \tilde{\mu} \tilde{\mu} F(x, \tilde{\mu})$

is the particle streaming in the wave frame. The next moment gives an equation

$\Gamma_{\mathrm{A}} \frac{\partial}{\partial x}\left[\tilde{V} \tilde{n}_{\|}(x)-V_{\mathrm{A}} \tilde{S}(x)\right]=-\frac{\tilde{S}}{\tau}$,

where

$\tilde{n}_{\|}(x)=\int_{-1}^{+1} \mathrm{~d} \tilde{\mu} \tilde{\mu}^{2} F(x, \tilde{\mu})$.

The function $F$ represents particles that have experienced at least one isotropizing scattering, so it is natural to make the diffusion approximation and replace $\tilde{n}_{\|}$by $\tilde{n} / 3$. This results in the pair of equations

$-V_{\mathrm{A}} \frac{\partial \tilde{n}}{\partial x}=\frac{\partial}{\partial x} \kappa(x) \frac{\partial \tilde{n}}{\partial x}+\frac{\tilde{V} Q}{\Gamma_{\mathrm{A}}\left(\tilde{V}+V_{\mathrm{A}}\right)}$,

and the modified Fick's law

$\tilde{S}=-\kappa \frac{\partial \tilde{n}}{\partial x}+V_{\mathrm{A}} \tau Q$

where the spatial diffusion coefficient is

$\kappa(x)=\frac{\left(\tilde{V}^{2}-3 V_{\mathrm{A}}^{2}\right) \Gamma_{\mathrm{A}} \tau(x)}{3}$.

We integrate the diffusion-convection equation once with the boundary condition $\partial \tilde{n} / \partial x \rightarrow 0$ at $x \rightarrow-\infty$ to get

$-\kappa \frac{\partial \tilde{n}}{\partial x}=\frac{\alpha_{2} \tilde{V} \tilde{n}_{\mathrm{i}}}{\alpha_{2}+1} \exp \left\{\int_{0}^{x} \frac{\mathrm{d} x^{\prime}}{\Gamma_{\mathrm{A}}\left(\tilde{V}+V_{\mathrm{A}}\right) \tau\left(x^{\prime}\right)}\right\}$

with

$\alpha_{2}=\frac{\tilde{V}^{2}-3 V_{\mathrm{A}}^{2}}{3 V_{\mathrm{A}}\left(\tilde{V}+V_{\mathrm{A}}\right)}$ 
Thus,

$$
\begin{aligned}
\tilde{S}(x) & =\tilde{n}_{\mathrm{i}}\left[\frac{\alpha_{2} \tilde{V}}{\alpha_{2}+1}+V_{\mathrm{A}}\right] \exp \left\{\int_{0}^{x} \frac{\mathrm{d} x^{\prime}}{\Gamma_{\mathrm{A}}\left(\tilde{V}+V_{\mathrm{A}}\right) \tau\left(x^{\prime}\right)}\right\} \\
& =\tilde{n}_{\mathrm{i}} \frac{\tilde{V}\left(\tilde{V}+V_{\mathrm{A}}\right)}{\tilde{V}+3 V_{\mathrm{A}}} \exp \left\{\int_{0}^{x} \frac{\mathrm{d} x^{\prime}}{\Gamma_{\mathrm{A}}\left(\tilde{V}+V_{\mathrm{A}}\right) \tau\left(x^{\prime}\right)}\right\} .
\end{aligned}
$$

The boundary condition for $\tilde{n}(x)$ at $x=0$ requires some care, if accurate results are to be obtained. We assume that the scatterings are efficient enough to keep the particles quasi-isotropic at the spatial boundary in the forward hemisphere, while the backward hemisphere is empty. This gives the ratio of streaming to the density close to $\tilde{V} / 2$. Thus, at $x=0$ we should use

$\tilde{n}_{0} \equiv \tilde{n}(0)=\frac{2 \tilde{S}(0)}{\alpha_{3} \tilde{V}} \equiv \frac{2 \tilde{S}_{0}}{\alpha_{3} \tilde{V}}=\frac{2\left(\tilde{V}+V_{\mathrm{A}}\right)}{\alpha_{3}\left(\tilde{V}+3 V_{\mathrm{A}}\right)} \tilde{n}_{\mathrm{i}}$,

where $1 \lesssim \alpha_{3}<2$ is a numerical constant close to unity. This gives immediately the particle flux out back to the upstream region as

$$
\begin{aligned}
\tilde{S}_{0}-V_{\mathrm{A}} \tilde{n}_{0} & =\frac{\alpha_{3} \tilde{V}-2 V_{\mathrm{A}}}{\alpha_{3} \tilde{V}} \tilde{S}_{0} \\
& =\frac{\alpha_{3} \tilde{V}-2 V_{\mathrm{A}}}{\alpha_{3}\left(\tilde{V}+3 V_{\mathrm{A}}\right)}\left(\tilde{V}+V_{\mathrm{A}}\right) \tilde{n}_{\mathrm{i}},
\end{aligned}
$$

which yields a transmission coefficient (omitting the subscript $j$ ) of

$T=1-\frac{\alpha_{3} \tilde{V}-2 V_{\mathrm{A}}}{\alpha_{3}\left(\tilde{V}+3 V_{\mathrm{A}}\right)}=\frac{\left(3 \alpha_{3}+2\right) V_{\mathrm{A}}}{\alpha_{3}\left(\tilde{V}+3 V_{\mathrm{A}}\right)} \approx \frac{3 \alpha_{3}+2}{\alpha_{3}} \frac{V_{\mathrm{A}}}{\tilde{V}}$,

where the approximation holds for $V_{\mathrm{A}} \ll \tilde{V}$. With this boundary condition, we can also integrate the zeroth moment equation as

$$
\begin{aligned}
& \tilde{n}(x)-\tilde{n}_{0}=\frac{\tilde{S}(x)-\tilde{S}_{0}}{V_{\mathrm{A}}}-\int_{0}^{x} \frac{Q(x)}{\Gamma_{\mathrm{A}} V_{\mathrm{A}}}=\frac{3\left(\tilde{V}+V_{\mathrm{A}}\right)}{\tilde{V}+3 V_{\mathrm{A}}} \tilde{n}_{\mathrm{i}} \\
& \times\left(1-\exp \left\{\int_{0}^{x} \frac{\mathrm{d} x^{\prime}}{\Gamma_{\mathrm{A}}\left(\tilde{V}+V_{\mathrm{A}}\right) \tau\left(x^{\prime}\right)}\right\}\right),
\end{aligned}
$$

which yields

$$
V_{\mathrm{A}} \tilde{n}(-\infty)=T\left(\tilde{V}+V_{\mathrm{A}}\right) \tilde{n}_{\mathrm{i}},
$$

as it should, of course. Note that the condition typically used in diffusion models for an absorbing boundary, $\tilde{n}_{0}=0$ or $\alpha_{3} \rightarrow \infty$, would indicate a non-physical low value, $T=3 V_{\mathrm{A}} /\left(\tilde{V}+3 V_{\mathrm{A}}\right)$, of the transmission coefficient. On the other hand, a value of $\alpha_{3}=1$ gives $T=5 V_{\mathrm{A}} /\left(\tilde{V}+3 V_{\mathrm{A}}\right)$, which is still very close to the value obtained with the pitch-angle diffusion model.

Although it does not affect the value of the transmission coefficient, it is also interesting to note, that we can obtain information about the spatial structure of the turbulence inside the downstream plasma in the relaxation time approximation. We get (see Appendix A)

$$
\begin{aligned}
-\frac{\mathrm{d} U}{\mathrm{~d} x} & =\Gamma_{\mathrm{A}} \Gamma m_{j}\left(\tilde{V} Q-\frac{\tilde{S}}{\tau}\right) \\
& =\Delta U \frac{\mathrm{d}}{\mathrm{d} x} \exp \left\{\int_{0}^{x} \frac{\mathrm{d} x^{\prime}}{\Gamma_{\mathrm{A}}\left(\tilde{V}+V_{\mathrm{A}}\right) \tau\left(x^{\prime}\right)}\right\},
\end{aligned}
$$

where

$\Delta U=2 \tilde{\Gamma} m_{j} \tilde{V} \Gamma_{\mathrm{A}}^{2} V_{\mathrm{A}} \tilde{n}_{\mathrm{i}} \frac{\tilde{V}+V_{\mathrm{A}}}{\tilde{V}+3 V_{\mathrm{A}}}$

is the increase in wave-energy density due to particle isotropization, i.e., $U(-\infty)=U_{0}+\Delta U$. Compared to the magnetic field energy density inside the outflow plasma, $U_{B}=$ $B^{2} / 8 \pi$, we get a numerical estimate (for $V_{\mathrm{A}} \ll \tilde{V} \approx c$ )

$\frac{\Delta U}{U_{B}}=\frac{4 \tilde{\Gamma} m_{j} \tilde{V} \tilde{n}_{\mathrm{i}}}{m_{\mathrm{p}} V_{\mathrm{A}} n_{b}} \approx 0.55 \frac{\Gamma_{2}^{2} n_{\mathrm{i} 0}^{*} m_{j}}{B_{0} n_{\mathrm{b} 8}^{1 / 2} m_{\mathrm{p}}}$,

where $\tilde{\Gamma} \approx \Gamma=\Gamma_{2} 10^{2}, n_{b}=n_{b 8} 10^{8} \mathrm{~cm}^{-3}, \tilde{n}_{\mathrm{i}} \approx n_{\mathrm{i}}=\Gamma n_{\mathrm{i}}^{*}=$ $\Gamma n_{\mathrm{i} 0}^{*} \mathrm{~cm}^{-3}$, and $B=B_{0} \mathrm{G}$.

Although it seems simple, solving Eq. (37) requires some care, because $\tau$ depends on $U(x)$. Starting from the quasi-linear scattering rate,

$v \equiv \frac{2 \tilde{D}_{\mu \mu}}{1-\tilde{\mu}^{2}}=\pi \Omega_{j} \frac{\left|k_{j}\right| \tilde{I}\left(k_{j}\right)}{\tilde{\Gamma} B^{2}}$,

and replacing $\left|k_{j}\right| \tilde{I}\left(k_{j}\right)$ by $\alpha_{4} 2 \pi U(x) / \Gamma_{\mathrm{A}}^{2}$, where $\alpha_{4}$ is a numerical constant, gives an estimate (Skilling 1975)

$\frac{1}{\tau}=\alpha_{4} \frac{\pi}{4 \Gamma_{\mathrm{A}}^{2}} \frac{\Omega_{j}}{\tilde{\Gamma}} \frac{U}{U_{B}}$

We have, thus, implicitly assumed that the waves generated by electrons and protons are decoupled from each other. This is a valid assumption in case of electrons only if resonance broadening of low-frequency waves is efficient enough to make scattering of protons by electron-resonant waves $\left(|\tilde{k}|>\left|\Omega_{e}\right| / \tilde{\Gamma} \tilde{V}\right)$ negligible.

We now want to calculate the spatial structure in the energy density of the waves, $U(x)$. For that purpose, we can make the change of variable from $x$ to

$\xi=-\int_{0}^{x} \frac{\mathrm{d} x^{\prime}}{\Gamma_{\mathrm{A}}\left(\tilde{V}+V_{\mathrm{A}}\right) \tau\left(x^{\prime}\right)}$

to get

$\frac{\mathrm{d} U}{\mathrm{~d} \xi}=-\Delta U \frac{\mathrm{d} \mathrm{e}^{-\xi}}{\mathrm{d} \xi}$

It is now easy to solve for $U(\xi)$ in the range $\xi \geq 0$ as

$U(\xi)=U_{0}+\Delta U\left(1-\mathrm{e}^{-\xi}\right)$.

We can then express $x$ as a function of $\xi$ as

$x=-\int_{0}^{\xi} \mathrm{d} \xi^{\prime} \Gamma_{\mathrm{A}}\left(\tilde{V}+V_{\mathrm{A}}\right) \tau\left(\xi^{\prime}\right)$. 


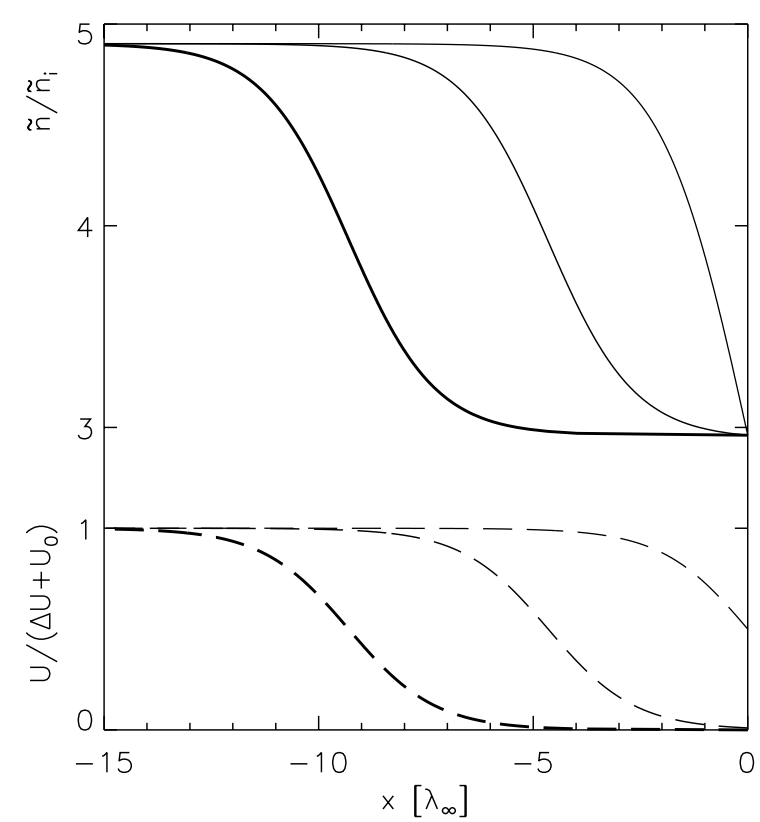

Fig. 3. Total particle density (solid curves) and wave-energy density (dashed curves) as a function of position for $V_{\mathrm{A}} / \tilde{V}=0.01, \alpha_{3}=1$, and three values of $\Delta U / U_{0}$ (from left to right) of $10^{4}, 100$, and 1 . The position is measured in asymptotic mean free paths (see Eq. (48)).

Plugging in $\tau(\xi)$ using Eqs. (41) and (44) gives an analytically integrable form for $x(\xi)$. The result is

$$
\begin{aligned}
x & =-\frac{4 \tilde{\Gamma}\left(\tilde{V}+V_{\mathrm{A}}\right)}{\alpha_{4} \pi \Omega_{j}} \frac{U_{B} \Gamma_{\mathrm{A}}^{3}}{U_{0}+\Delta U}\left[\xi+\ln \frac{U_{0}+\Delta U\left(1-\mathrm{e}^{-\xi}\right)}{U_{0}}\right] \\
& =\Gamma_{\mathrm{A}}\left(\tilde{V}+V_{\mathrm{A}}\right) \tau(\infty)\left[\xi+\ln \frac{U_{0}+\Delta U\left(1-\mathrm{e}^{-\xi}\right)}{U_{0}}\right] .
\end{aligned}
$$

This, together with Eq. (44), gives the solution for $U(x)$ in a parametric form. We note also that the total particle density as measured in the wave frame (including the beamed source) can also be given in the same form by

$\tilde{n}(\xi)=\tilde{n}_{\mathrm{i}} \frac{\left(3 \alpha_{3}+2\right)\left(\tilde{V}+V_{\mathrm{A}}\right)-2 V \mathrm{e}^{-\xi}}{\tilde{V}+3 V_{\mathrm{A}}}$.

This solution is plotted in Fig. 3.

We have, thus, solved the (non-linear) steady-state pick-up problem analytically in the relaxation-time approximation. Our results show that in this case the medium downstream of the relativistic collision front is capable of rather rapid isotropization of the incoming ions, since the mean free path is (for a non-relativistic $V_{\mathrm{A}}$ and $U_{0} \ll \Delta U$ )

$\lambda=3 \frac{\kappa}{\tilde{V}} \approx \tilde{V} \tau \stackrel{x \rightarrow-\infty}{\Longrightarrow} \frac{4 \tilde{\Gamma} \tilde{V}}{\alpha_{4} \pi \Omega_{j}} \frac{U_{B}}{\Delta U} \equiv \lambda_{\infty}$
$\lambda_{\infty} \approx \frac{V_{\mathrm{A}}}{\alpha_{4} \pi \Omega_{\mathrm{p}}} \frac{n_{b}}{n_{\mathrm{i}}} \approx 7.2 \times 10^{8} \frac{n_{\mathrm{b} 8}^{1 / 2}}{\alpha_{4} \Gamma_{2} n_{\mathrm{i} 0}^{*}} \mathrm{~cm}$.

Note that our calculated mean free path is smaller than that of Pohl \& Schlickeiser (2000) by a large fraction of $3 \ln \left(c / \sqrt{2} r_{I}^{2} V_{\mathrm{A}}\right)$ that mainly results from our assumption of efficient resonance broadening. Here, $r_{I}$ measures the ratio of background turbulence intensity to the self-generated one (see Appendix B).

Although the mean free path is the scale of isotropization, it is not the length scale determining the particle confinement in the downstream medium of a relativistic collision front. The assumption of an infinite extent of the downstream region made in our calculation is only valid if the diffusion length $L_{\mathrm{D}}=\kappa / V_{\mathrm{A}}$ is much smaller than the actual extent of the downstream region (Ostrowski \& Schlickeiser 1996; Vainio et al. 2000). The diffusion length is numerically given by

$L_{\mathrm{D}} \approx \frac{c}{3 \alpha_{4} \pi \Omega_{\mathrm{p}}} \frac{n_{b}}{n_{\mathrm{i}}} \approx 3.3 \times 10^{11} \frac{n_{b 8}}{\alpha_{4} B_{0} \Gamma_{2} n_{\mathrm{i} 0}^{*}} \mathrm{~cm}$.

For typical parameter values and $\alpha_{4} \sim 1$ our model is still valid, but clearly there could be cases when we should consider the effects of a finite downstream region, which would increase the transmission and reduce the flux of particles back to the upstream medium. In this case, however, the solution can be calculated with a constant mean free path $\lambda=\lambda_{\infty}$, because unless $\Delta U / U_{0}$ is enormous, the distance over which the mean free path attains its final value is less than the diffusion length. Solving the finite-extent problem including the energyloss effects, i.e., for radiative conditions, will be the subject of a forth-coming paper.

\subsection{Discussion of the modeled wave energy densities}

Let us compare the results of the quasi-linear and relaxation time models. We can estimate the self-generated wave-energy density obtained from the quasi-linear model by using the simplest possible assumption, an isotropic boundary density at $\tilde{\mu}>V_{\mathrm{A}} / \tilde{V}$. Taking $T_{j}=\alpha_{1} 4 \tilde{V} V_{\mathrm{A}} /\left(\tilde{V}+V_{\mathrm{A}}\right)^{2}$ we get, after a lengthy but straight-forward calculation, a self-generated waveenergy density of

$$
\begin{aligned}
& \frac{\Gamma_{\mathrm{A}}^{2}}{4 \pi} \int \mathrm{d} \tilde{k}\left[\tilde{I}(\tilde{k},-\infty)-I_{0}(\tilde{k})\right] \\
& \quad=\sum_{j=e, p} \Gamma_{\mathrm{A}}^{2} \tilde{\Gamma} m_{j} \int_{-1}^{+1} \mathrm{~d} \tilde{\mu} \tilde{V} \tilde{\mu}\left(\tilde{V} \tilde{\mu}-V_{\mathrm{A}}\right)[F(0, \tilde{\mu})-F(-\infty)] \\
& \quad=\sum_{j=e, p} \Gamma_{\mathrm{A}}^{2} \tilde{n_{\mathrm{i}}} \tilde{\Gamma} m_{j} \frac{\left[\left(5-4 \alpha_{1}\right) \tilde{V}+V_{\mathrm{A}}\right]\left(\tilde{V}+V_{\mathrm{A}}\right)}{3}
\end{aligned}
$$

This equals $\Delta U$ for the specific selection of

$\frac{\left[\left(5-4 \alpha_{1}\right) \tilde{V}+V_{\mathrm{A}}\right]}{3}=\frac{2 V_{\mathrm{A}} \tilde{V}}{\tilde{V}+3 V_{\mathrm{A}}}$

giving

$\alpha_{1}=\frac{5\left(\tilde{V}+V_{\mathrm{A}}\right)^{2}-2 V_{\mathrm{A}}^{2}}{4 \tilde{V}\left(\tilde{V}+3 V_{\mathrm{A}}\right)}$

or $T \approx 5 V_{\mathrm{A}} /\left(\tilde{V}+3 V_{\mathrm{A}}\right)$ for $V_{\mathrm{A}} \ll \tilde{V}$ consistent with the result of the diffusion model for $\alpha_{3}=1$. This, of course, would require quite a large background spectrum, $r_{I}=\alpha_{1}-1$ (see Appendix B), to be consistent with the requirement of the positive wave spectrum in the quasi-linear model with $\tilde{I}_{0}(\tilde{k}) \propto \tilde{k}^{-2}$. 
We note that the wave-energy density is extremely sensitive to the value of the transmission coefficient: an increase of $\alpha_{1}$ from 1 to 1.25 leads to the decrease of the wave-energy density to a fraction of $V_{\mathrm{A}} /\left(\tilde{V}+V_{\mathrm{A}}\right)$ of the original value. Finally, we note that this sensitiveness to the parameters makes the model self-regulating: we can not propose an enlargement of this parameter much above $\alpha_{1}=1.25$ without making the forward waves unstable at least in part of the resonant wavenumber range. Generation of forward waves would make the resonant particles convect with them toward the edge of the blob. This automatically rules out the possibility of a large $\alpha_{1}$, except in a situation, where the resonance gap is present.

Let us compare the wavenumbers generated by the relativistic protons to the typical macroscopic scales of the model. Our parameters give

$k^{-1} \lessgtr \frac{\Gamma c}{\Omega_{\mathrm{p}}} \approx 3.1 \times 10^{8} \frac{\Gamma_{2}}{B_{0}} \mathrm{~cm}$.

Compared to the macroscopic scales, $d \gtrsim 10^{12} \mathrm{~cm}$ and $R \gtrsim$ $10^{13} \mathrm{~cm}$, the wave lengths are clearly orders of magnitude smaller and lie between the macroscopic stirring scales and the dissipative scales, i.e., in the inertial range of the background turbulence. We have, however, modeled the turbulence including no spectral energy transfer of the waves. At first, this might seem to be in conflict with the large energy densities obtained, but this is actually not the case: since the modeled wave field is unidirectional there should be very little interaction between the wave packets and, thus, the assumptions are internally consistent.

\section{Discussion of the results and limitations of the spatial modeling}

In the preceding sections we have investigated the spatial structure of ultra-relativistic collision-less collision fronts in the non-radiative limit by two different methods. Though the merits and shortcomings of the two techniques used are different, the results are very similar, and thus deserve confidence.

The main result is that most of the upstream particles, that traverse the collision front, will eventually be reflected, for their isotropization occurs when they have propagated a distance corresponding to a few scattering mean free paths in the downstream plasma. Once isotropized, the particles as an ensemble convect away from the boundary with Alfvén speed $V_{\mathrm{A}} \ll c$, while individually they can quickly reach the boundary by random-walk over the few scattering mean free paths. We find the mean free path for scattering independent of the particles' energy (see Eq. (49)). Our study therefore indicates, that the isotropization length scale is similar to the effective thickness of the collision front for all particle energies, and that thus relativistic particles will never see the collision front as a perfect hydrodynamical shock. Then, particle acceleration at parallel, ultra-relativistic shocks would be less efficient than previously modeled assuming discontinuous shocks.

A second result, following from the first, is that the pick-up particles spend less time in the dense downstream medium, where they can radiate. The consequences for our understanding of GRBs and AGNs are potentially large. Most GRB models are not very specific in their treatment of the energy transfer from the relativistic protons to the electrons in the downstream medium, and we therefore find it difficult to quantify the effects on the radiation output in the context of a published scenario. We may, however, discuss in the next section, to what extent the predictions of Pohl \& Schlickeiser (2000) for the radiation products of the relativistic protons in the jets of AGNs need to be modified.

Before we turn to that, we will qualitatively discuss two limitations of our treatment and their possible impact on the results. First, the reflected particles may interact with the upstream medium, thus heating and repelling the interstellar medium. The question is to what extent that would modify or possibly impede the transfer of interstellar particles to the downstream region. Second, our calculation are based on the assumption of cold media, and we have to explore the possible effects of the finite temperatures on our results. Let us discuss both in turn.

\subsection{Reflected particles in the upstream region}

If the Alfvén speed at the pick-up distance inside the outflow plasma is not close to the speed of light, the reflected particle flux can be almost equal to the incident particle flux. In this case, we have to ask what happens in the upstream region $(x>0)$, where the reflected particles meet the cold ambient medium. Since both components have an almost equal flux in the rest frame of the outflow, while the velocities are similar, it is not possible to treat the reflected particles with a quasi-linear approximation. Nevertheless, the mixture should be highly unstable and it should form a relativistic plasma through a number of transverse and electrostatic instabilities.

The question is whether or not these processes are rapid enough to significantly decelerate the interstellar particles in the outflow frame. If that is not the case, the interstellar particles would still enter the outflow region, but not necessarily as a cold beam. In the next subsection we will discuss the possible modifications arising from the ambient plasma having a finite temperature in the outflow frame.

If the interstellar particles are significantly decelerated in the outflow frame, a bow wave should form, that might collapse to a second relativistic collision front. The outflow plasma may act almost as a rigid piston pushing through the ambient gas and converting the collimated kinetic energy to random, thermal motion. The second collision front would impede the propagation of interstellar particles to the dense outflow plasma, and thus limit the flux of reflected particles that feed the bow wave. Therefore the physical status of the upstream plasma will be given by a balance between the kinetic interaction rates applicable to the situation, the details of which are beyond the scope of the present paper.

In a hydrodynamical description, there are, in principle, two possibilities: the bow shock can either run away from the piston (the collimated outflow) or it can be stationary, depending on the properties of the relativistic gas between the piston and the shock. In either case, the shock is ultra-relativistic and strong, 
has an ultra-relativistic downstream gas, and has its normal parallel to the ambient magnetic field. In the frame, where the shock is stationary, the gas just downstream the shock, therefore, flows at speed

$v_{2}=\frac{c}{3}$,

has a fluid-frame density of

$n_{2}=\Gamma_{1} \sqrt{8} n_{\mathrm{i}}^{*}$,

and a pressure of

$p_{2}=\frac{2}{3} \Gamma_{1}^{2} m_{\mathrm{p}} n_{1}^{*} c^{2}$

where $\Gamma_{1}$ is the Lorentz factor of the upstream flow relative to the shock (e.g., Granot \& Königl 2001). The downstream fluid near the piston would flow at the (piston's) Alfvén speed $V_{\mathrm{A}}$ relative to the piston toward it. If the state of the gas is conserved through the whole sheath region (i.e., if the gas is not substantially heated or cooled within the sheath), we get $\Gamma_{1}=\sqrt{2} \tilde{\Gamma}$, giving $n_{2}=4 \tilde{n}_{\mathrm{i}}$ and $p_{2}=4 \tilde{\Gamma} m_{\mathrm{p}} \tilde{n}_{\mathrm{i}} c^{2} / 3$. Thus, the protons entering the piston and producing radiation would be quasi-isotropic and have a large temperature, $\tilde{\Gamma} m_{\mathrm{p}} c^{2}$, corresponding to randomization of the propagation directions of the incoming particles. Radiation out from the dense outflow plasma would, thus, be at similar levels as without the shock, since the flux of particles into the blob, $\approx 4 V_{\mathrm{A}} n_{\mathrm{i}}$, and the mean proton Lorentz factor, $\approx \Gamma$, are of the same order in both cases.

\subsection{The effects of finite temperatures}

Throughout this study, we have assumed for simplicity that both the outflow plasma plasma and the ambient interstellar medium are cold, implying that Alfvén waves are undamped by the plasma particles. For a finite temperature, damping of the waves by the cyclotron resonance would occur with a resonance condition $\tilde{k}=-\Omega_{\mathrm{p}} /\left(\tilde{\Gamma} \tilde{v}_{\|}\right)$, where $\tilde{\Gamma} \tilde{v}_{\|}$is the parallel component of the proper velocity of thermal protons. As long as the proton temperature is much smaller than the kinetic energy of the incoming interstellar particles, $k_{B} T_{p, b} \ll(\Gamma-1) m_{\mathrm{p}} c^{2}$, the thermal particles of the downstream medium resonate with (and damp) only a minor part of the wavenumber spectrum interacting with the relativistic particles. Work in progress suggests that this is indeed the case (Siewert et al. in prep.).

The assumption of the ambient plasma being cold simplifies the treatment of wave generation in the downstream region considerably, but is actually inconsistent with the finding of Pohl et al. (2002) that the electrostatic instabilities inside the outflow plasma are typically faster than the electromagnetic ones. This means that prior to isotropization, the mono-energetic interstellar particle distribution is changed to a plateau distribution, i.e., the kinetic temperature of the pickedup particles is actually of the same order as the ordered kinetic energy. A similar situation could be caused by shock formation ahead of the ejecta (see above). The effects of the plateaued particle distribution to the spectrum of electromagnetic waves were studied by Schlickeiser et al. (2002), and found to be very minor. The effects on the $\gamma$-radiation were also found minor by Pohl et al. (2002), so the results presented here are qualitatively correct. Thus, unless the temperature in the ambient medium is larger than the kinetic energy of the blast-wave particles, $T_{p, i}>(\Gamma-1) m_{\mathrm{p}} c^{2}$ (which we have no reason to assume), a finite temperature of the ambient medium has no qualitative effect on our results.

In the quasilinear description, the damped waves lead to resonance broadening giving particles a way to cross the region close to $\tilde{\mu}=0$ (Schlickeiser \& Achatz 1993), but estimating the quantitative effects on the mean free path is out of the scope of the present analysis, since the details of scattering across the QLT resonance gaps were anyway left unspecified in this paper.

\section{Radiation modeling of relativistic outflows}

Let us now discuss the modifications, that our results on the spatial transport of picked-up particles impose on the radiation modeling of AGNs and GRBs. For that purpose, we will use the AGN model of Pohl \& Schlickeiser (2000) as an example.

In that scenario the temporal evolution of picked-up particles is determined by a continuity equation for the number spectrum of energetic protons, $N_{\mathrm{p}}(\gamma)$, in the outflow frame

$$
\frac{\partial N_{\mathrm{p}}}{\partial t}+\frac{\partial}{\partial \gamma}\left(\dot{\gamma} N_{\mathrm{p}}\right)+\frac{N_{\mathrm{p}}}{T_{\mathrm{E}}}+\frac{N_{\mathrm{p}}}{T_{\mathrm{N}}}=\dot{N}_{\mathrm{p}},
$$

where $\dot{\gamma}$ is the proton energy-loss rate (elastic+inelastic), $T_{\mathrm{E}}=$ $d^{2} / \kappa$ is the escape time of particles out from the blob, $T_{\mathrm{N}}$ is the time scale for neutron escape after $(p \rightarrow n)$ reactions, and

$\dot{N}_{\mathrm{p}}=\pi R^{2} c n_{\mathrm{i}}^{*} \sqrt{\Gamma^{2}-1} \delta(\gamma-\Gamma)$,

is the rate of relativistic proton injection by isotropization of the incoming interstellar particles. The spatial transport effects discussed in this paper have an influence on $\dot{N}_{\mathrm{p}}$ and $T_{\mathrm{E}}$. All other parameters are taken as in Pohl \& Schlickeiser (2000).

In contrast to Pohl \& Schlickeiser (2000), we need to consider the reflected and transmitted particles separately. A fraction of $T_{j}$ of the injection rate $\dot{N}_{\mathrm{p}}$ will be transmitted and a fraction of $1-T_{j}$ back-scattered. We will assume a nonrelativistic $V_{\mathrm{A}}$. In this case the protons will be almost isotropic in the outflow frame and lose a negligible fraction of their energy during the isotropization process.

During the calculation of the transmission coefficient, we assumed that the diffusion length is small compared to the thickness of the outflow plasma (and showed a-posteriori this assumption to be reasonable). Making the same assumption here, we get the escape time of transmitted particles by convection as

$$
T_{\mathrm{ET}}=\frac{d}{V_{\mathrm{A}}}=4.6 \times 10^{4} \frac{d_{12} n_{b 8}^{1 / 2}}{B_{0}} \mathrm{~s},
$$

where $d=d_{12} 10^{12} \mathrm{~cm}$. For comparison, the time scale for diffusive propagation to the backside of the outflow plasma is

$T_{\mathrm{ED}}=\frac{3 d^{2}}{c \lambda_{\infty}} \simeq 1.4 \times 10^{5} \frac{d_{12}^{2} n_{\mathrm{i} 0}^{*} \Gamma_{2}}{n_{b 8}^{1 / 2}} \mathrm{~s}$.

The mean residence time of reflected particles inside the blob can be calculated applying standard results of diffusion theory, 

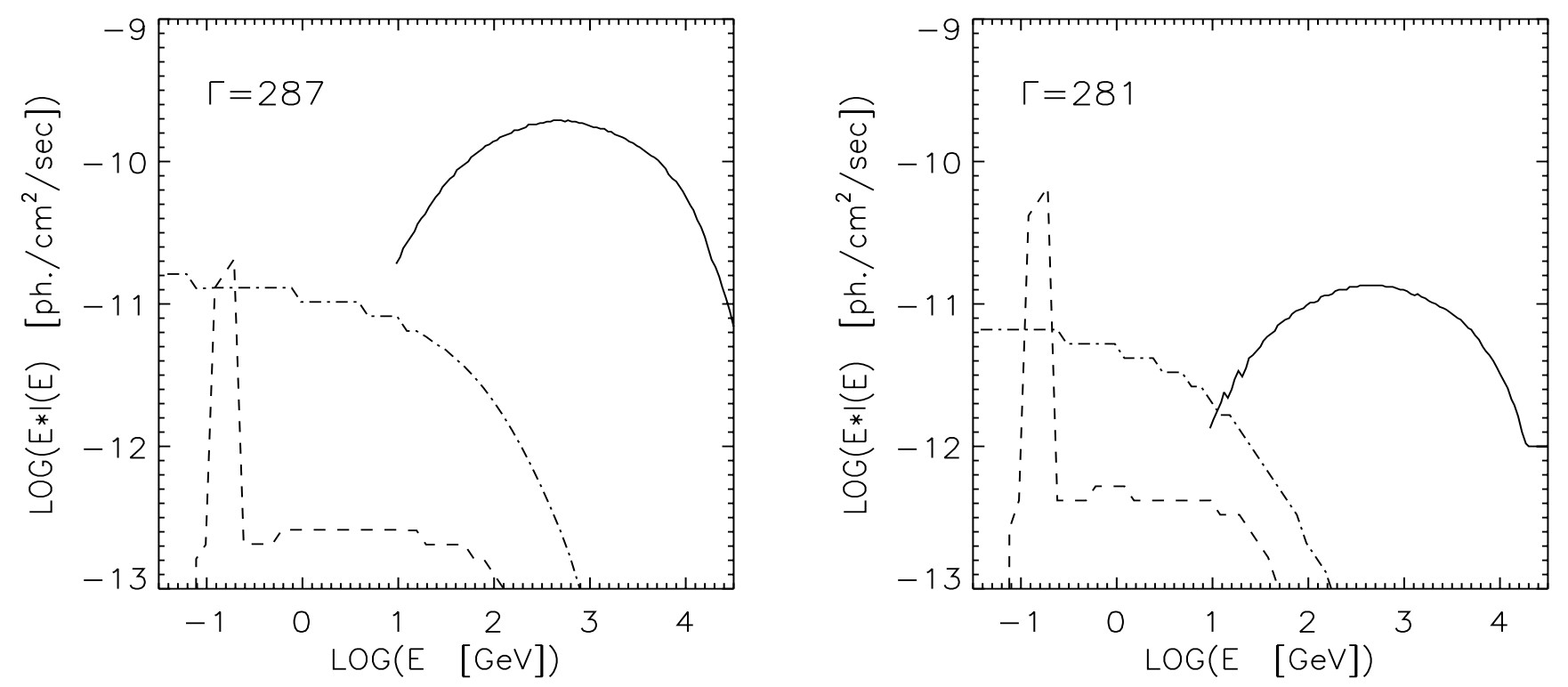

Fig. 4. Gamma radiation from a relativistic outflow with $n_{b}=10^{9} \mathrm{~cm}^{-3}, R=10^{14} \mathrm{~cm}, d=10^{13} \mathrm{~cm}$ and $\Gamma(t=0)=300$ propagating into a medium with $n_{\mathrm{i}}^{*}=0.1 \mathrm{~cm}^{-3}$ and $B=5 \mathrm{G}$ viewed at an aspect angle of $0.1^{\circ}$ and $t=1 \mathrm{~h}$ observer's time. The bulk Lorentz factor at that time is indicated in each panel. The components shown result from $\pi^{0}$-decay (solid curves), positron annihilation (dashed curves) and bremsstrahlung (dot-dashed curves). The left-hand and right-hand panels show the results for the original model of Pohl \& Schlickeiser (2000) and for the present model, respectively.

like in case of calculating the mean residence time of particles downstream of a non-relativistic shock wave (see, e.g., Drury 1983). It is given by

$T_{\mathrm{ER}}=\frac{4 L_{\mathrm{D}}}{c} \approx 44 \frac{n_{b 8}}{\alpha_{4} B_{0} \Gamma_{2} n_{\mathrm{i} 0}^{*}} \quad \mathrm{~s}$.

The time scale of pion production inside the dense outflow plasma was given by Pohl \& Schlickeiser (2000) for ultrarelativistic proton energies as

$\tau_{\pi} \approx 1.4 \times 10^{7} n_{b 8}^{-1} \quad \mathrm{~s}$.

Thus, for typical blast-wave parameters, the escape times are short compared to the pion-production time scale, which is of the same order as the neutron-escape time. In this case, the steady-state spectrum of the radiating relativistic protons inside the dense downstream plasma is

$$
\begin{aligned}
N_{\mathrm{p}} & \approx\left[\left(1-T_{\mathrm{p}}\right) T_{\mathrm{ER}}+T_{\mathrm{p}} T_{\mathrm{ET}}\right] \dot{N}_{\mathrm{p}} \\
& \simeq\left[\left(1-T_{\mathrm{p}}\right) \frac{4 L_{\mathrm{D}}}{c}+T_{\mathrm{p}} \frac{d}{V_{\mathrm{A}}}\right] \pi R^{2} c n_{\mathrm{i}}^{*} \sqrt{\Gamma^{2}-1} \delta(\gamma-\Gamma) .
\end{aligned}
$$

The first term is included for completeness, but it can be neglected for typical parameters and $T_{\mathrm{p}}=5 V_{\mathrm{A}} / c$. Thus, the result is in accordance with the density obtained from the relaxationtime approximation (see Eq. (36)).

Comparing with the typical, diffusive-escape dominated $\left(\tau_{\pi} \gg T_{\mathrm{ED}}=d^{2} / \kappa\right)$ solution of Pohl \& Schlickeiser (2000), $N_{\text {PS }}$, with their scattering mean free path

$\lambda_{\mathrm{PS}} \simeq 6 \times 10^{10} \frac{n_{b 8}^{1 / 2}}{\Gamma_{2} n_{\mathrm{i} 0}^{*}} \mathrm{~cm}$ we note, that the density of energetic protons calculated in this paper is lower than that derived by studying the temporal evolution only:

$N_{\mathrm{p}}=\frac{T_{\mathrm{p}} c \lambda_{\mathrm{PS}}}{3 V_{\mathrm{A}} d} N_{\mathrm{PS}} \simeq 0.1 \frac{n_{\mathrm{b} 8}^{1 / 2}}{d_{12} \Gamma_{2} n_{\mathrm{i} 0}^{*}} N_{\mathrm{PS}}$.

Thus the spatial-transport effects will produce about an order of magnitude decrease in the luminosity for a fixed set of model parameters, provided that the transport of secondary electrons and positrons is radiation dominated. That was not always the case in the treatment of Pohl \& Schlickeiser (2000), but is here, for we have derived a significantly smaller scattering length and thus the time scale for the escape of transmitted particles is substantially longer than in the earlier calculation. As we will see later, the spectra of leptonic emission are therefore more evolved toward a cooling distribution than was the case in the original paper.

The reflection of particles also affects the deceleration of the outflow, as now the momentum deposited in the outflow plasma by the reflected particles is $50 \%$ higher than before, when the back-scattering of particles was neglected. The timescale for slowing down the outflow is, thus, decreased by a factor of $\approx 2 /\left(3-T_{j}\right)$ relative to the modeling of Pohl \& Schlickeiser (2000). The faster deceleration also affects the luminosity at a given (observer's) time since the Lorentz factor enters both the proton source function and the Doppler factor of the outflow.

We have modified the code of Pohl \& Schlickeiser (2000) to take account for the spatial transport effects, as described above. In Fig. 4, we present the gamma radiation emitted by a relativistic outflow (ejected from and AGN at redshift $z=0.5$ ) with a number density $n_{b}=10^{9} \mathrm{~cm}^{-3}$, radius $R=10^{14} \mathrm{~cm}$, thickness $d=10^{13} \mathrm{~cm}$ and an initial Lorentz factor of $\Gamma=300$ 
propagating through a medium with a density $n_{\mathrm{i}}^{*}=0.1 \mathrm{~cm}^{-3}$ and magnetic field of $B=5 \mathrm{G}$, viewed at an aspect angle of $0.1^{\circ}$. We take $T_{j}=4 V_{\mathrm{A}} c /\left(c+V_{\mathrm{A}}\right)^{2}$ and neglect radiation from the reflected particles. The time of the observation is 1 hour (observer's time) after the emission of the outflow plasma. The spectral components shown result from $\pi^{0}$-decay, bremsstrahlung from the secondary electrons and positrons and the annihilation radiation from secondary positrons. As expected, the $\pi^{0}$-decay component shows a large decrease due to spatial effects, but not so the radiation signatures of mildly relativistic leptons, for these particles have a higher probability to cool down from their initial high Lorentz factors.

About one order of magnitude in $\mathrm{TeV}$ radiation efficiency is lost due to the reduced number of relativistic protons in the dense outflow plasma, and another factor of two is the result of the enhanced deceleration. As many of the parameters involved in the model are unknown, most notably radius, $R$, of the outflow front, we can not make a quantitative prediction for gamma-ray flux from a given object. It is likely, that a distribution exists for the spatial extent of the outflow plasma as well as for its bulk Lorentz factor. All we can say is that the corresponding distribution in $\mathrm{TeV}$-scale gamma-ray flux or apparent luminosity is shifted by approximately a factor of thirty toward lower fluxes. The TeV blazars observed to date are located at redshifts not exceeding 0.15 with a typical flux of $10^{-11} \mathrm{ph} . / \mathrm{cm}^{2} / \mathrm{s}$, and thus correcting for the redshift their apparent luminosity is lower than that shown in Fig. 4 even accounting for the spatial transport effects.

While the calculated proton spectra are close to monoenergetic, as predicted by the analytical approximation, they still create rather broad pion and secondary electron spectra, as can be deduced from the components of the computed $\gamma$-ray spectrum, because even mono-energetic protons produce a broad spectrum of pions. This suggests that order-of-magnitude type estimates of pion energies being a fixed fraction of proton energy may be dangerous and lead to incomplete conclusions about the importance of hadronic radiation processes at different $\gamma$-ray energies. Note, however, that our modeled spectral shape should not be taken as a prediction of gamma ray spectrum in the sub-GeV range, because no IC emission was included. Accurate modeling of this component is out of the scope of the present paper because it requires one to specify the ambient photon field. One can, however, give a rough estimate for it by neglecting the Klein-Nishina modification of the Compton cross section. In that case, the spectral form of the IC component would be similar to that of the synchrotron spectrum (see Fig. 4 of Pohl \& Schlickeiser 2000), but it would be found in the $\mathrm{MeV}-\mathrm{GeV}$ range rather than in the eV-keV range in case of synchrotron emission. The IC emission could well dominate the spectrum at sub-GeV $\gamma$-ray energies, and the spectral shape in the EGRET energy range could be close to flat in $v F_{v}$, as observed for BL Lacs. Note also that a comparison of the model with observational data requires one to integrate the predicted spectrum over the integration time of the observing instrument. Since our model predicts possibly fast-shifting peaks (due to a strong time dependence of the Doppler factor), the modeled spectrum may have even broader components than the presented snapshot gamma-ray spectra.

\section{Summary and outlook}

We have investigated the interaction between a collimated relativistic outflow and ambient medium through a collisionless collision front in kinetic theory. Using steady-state particle transport equations, we have demonstrated that by picking up ambient particles through the generation of low-frequency transverse plasma waves, the outflow is capable of producing an isotropic particle distribution inside the dense downstream plasma provided that the resonance gap (at $\tilde{\mu}=0$ ) of the selfgenerated Alfvénic turbulence is filled. A major fraction of the incident particle flux would, however, be backscattered in a steady state: the transmitted particle flux would be a small fraction $T_{j}=(4-5) \times V_{\mathrm{A}} / c$ (for a non-relativistic $V_{\mathrm{A}}$ ) of the incident particle flux.

Our findings have consequences for the modeling of relativistic shock acceleration and for the radiation modeling of GRBs and AGNs. Our results imply that swept-up energetic particles spent less time than previously thought in the dense downstream plasma, where they can efficiently radiate. As a particular example, we have discussed the impact on the gamma-ray spectra in the AGN model of Pohl \& Schlickeiser (2000).

We made our calculations assuming that the thickness of the outflow plasma is much larger than the diffusion length of the particles in the downstream region. This assumption simplifies the calculation of the transmitted flux by allowing us to neglect the escape of particles by diffusion when compared to the escape by convection at Alfvén speed. The assumption has to be taken into account also when determining the escape time of particles in the radiation model. Since the typical residence time of the reflected particles is very small compared to the Alfvénic escape time of the transmitted particles, the reflected particles do not significantly contribute to the radiation from the dense downstream plasma. We found that for fixed parameters in the typical range, the number of radiating protons inside the collimated outflow is down by an order of magnitude in the present model relative to that in purely temporal studies. However, the modeled luminosity can be increased by increasing the dimensions of the outflow plasma.

Without efficient particle scattering across $\mu=0$, in which case no flux is reflected $\left(T_{j}=1\right)$, one has to remodel the electromagnetic emission using particle distributions that are isotropic only in the backward hemisphere $(\tilde{\mu}<0)$ and take into account that particles now traverse the outflow plasma and escape quite rapidly compared to the isotropized case (in $2 d / c$ vs. $d / V_{\mathrm{A}}$ for the two cases, respectively).

We emphasize the need for thorough kinetic studies of the interaction between the highly relativistic reflected particles and the thermal upstream medium of the collisionless collision front. In the case of strong back-scattering, the incident and reflected particles may form a relativistic plasma ahead of the ejecta, that would substantially modify the structure of the collision front and may impede the transport of relativistic particles to the dense downstream medium.

All calculations presented in this paper need to be modified for outflows that are thin compared to the diffusion length. One effect of a finite thickness should be the reduction of the 
reflected particle flux. The luminosity will also be decreasing since the reflected particle flux is only smaller because the escaping particle flux gets larger: instead of being reflected, the particle can now propagate quickly to the other side of the downstream region and escape from there before producing radiation. The quantitative analysis of the effect of the finite thickness will be a subject of a forthcoming study.

To calculate the mean free paths accurately in the quasilinear approximation, one would have to include forward waves and/or specify the background-wave spectra. One should also include electrostatic waves in the calculation. Eventually, one has to drop the assumption of a steady state and to address the problem in a time-dependent manner. This can probably only be done via numerical simulations, for which a number of tools are already under development (see, e.g., Vainio \& Kocharov 2001; Vainio 2002).

Acknowledgements. This work is part of the project "Collective Processes in Astrophysical Plasmas: Waves, Heating and Accelerated Particles" co-funded by the Academy of Finland and DAAD. R.V. also acknowledges the financial support of the Academy of Finland, project 46331, and the EU training network PLATON, EC contract HPRN-CT-2000-00153. MP and RS acknowledge support by the Verbundforschung, grant DESY CH1PCA6.

\section{References}

Achterberg, A., Gallant, Y. A., Kirk, J. G., \& Guthmann, A. W. 2001, MNRAS, 328, 393

Bednarz, J., \& Ostrowski, M. 1996, MNRAS, 283, 447

de Hoffmann, F., \& Teller, E. 1950, Phys. Rev., 80, 692

Drury, L. O'C. 1983, Rep. Prog. Phys., 46, 973

Ellison, D. C., Baring, M. G., \& Jones, F. C. 1996, ApJ, 473, 1029

Ellison, D. C., \& Reynolds, S. P. 1991, ApJ, 378, 214
Ellison, D. C., \& Double, G. P. 2002, Astrop. Phys., 18, 213

Gaidos, J. A., Akerlof, C. W., Biller, S. D., et al. 1996, Nature, 383, 318

Granot, J., \& Königl, A. 2001, ApJ, 560, 145

Henri, G., Pelletier, G., Petrucci, P. O., \& Renaud, N. 1999, APh, 11, 347

Homan, D. C., Wardle, J. F. C., Cheung, C. C., et al. 2002, ApJ, 580, 742

Homan, D. C., Lister, M. L., Kellermann, K. I., et al. 2003, ApJ, 589, L9

Kirk, J. T., Schlickeiser, R., \& Schneider, P. 1988, ApJ, 328, 269

Kirk, J. G., \& Duffy, P. 1999, J. Phys. G, 25, R163

Kirk, J. G., Guthmann, A. W., Gallant, Y. A., \& Achterberg, A. 2000 ApJ, 542, 235

Metzger, M. R., Djorgovski, S. G., Kulkarni, S. R., et al. 1997, Nature, 387, 87

Ostrowski, M., \& Schlickeiser, R. 1996, Sol. Phys., 167, 381

Pelletier, G. 1999, A\&A, 350, 705

Pohl, M., \& Schlickeiser, R. 2000, A\&A, 354, 395

Pohl, M. 2002, in Proc. 27th Int. Cosmic Ray Conf., Invited, rapporteur, and highlight papers, Copernicus Gesellschaft, ed. R. Schlickeiser, 147

Pohl, M., Lerche, I., \& Schlickeiser, R. 2002, A\&A, 383, 309

Schlickeiser, R., \& Achatz, U. 1993, J. Plasma Phys., 49, 63

Schlickeiser, R., Vainio, R., Böttcher, M., et al. 2002, A\&A, 393, 69

Schneider, P., \& Kirk, J. G. 1987, ApJ, 323, L87

Skilling, J. 1975, MNRAS, 173, 255

Vainio, R. 2000, ApJS, 131, 519

Vainio, R. 2002, in High Energy Blazar Astronomy, ed. L. O. Takalo, \& E. Valtaoja, ASP Conf. Ser., 299, 143

Vainio, R., Kocharov, L., \& Laitinen, T. 2000, ApJ, 528, 1015

Vainio, R., \& Laitinen, T. 2001, A\&A, 371, 738

Vainio, R., \& Kocharov, L. 2001, A\&A, 375, 251

Vainio, R., \& Schlickeiser, R. 1998, A\&A, 331, 793

Vainio, R., \& Schlickeiser, R. 1999, A\&A, 343, 303

Vainio, R., Virtanen, J. J. P., \& Schlickeiser, R. 2003, A\&A, 409, 821

Vermeulen, R. C., \& Cohen, M. H. 1994, ApJ, 430, 467 
R. Vainio et al.: Particle transport effects in relativistic collision fronts, Online Material p 1

\section{Online Material}




\section{Appendix A: Wave equation and growth rate}

In this Appendix, we use again the notation, where the quantities measured in the wave frame are denoted with tilde. Let us write down an equation for the Alfvén wave propagation parallel to the magnetic field in the blob frame. The waves have a dispersion relation

$\omega=-k V_{\mathrm{A}}+i \sigma(k, x) / 2$,

where

$V_{\mathrm{A}}^{2}=\frac{v_{\mathrm{A}}^{2}}{1+v_{\mathrm{A}}^{2} / c^{2}} ; \quad v_{\mathrm{A}}^{2}=\frac{B^{2}}{4 \pi m_{\mathrm{p}} n_{b}}$

so the blob-frame wave intensity, $I(k, x) \mathrm{d} k=\left(\delta B_{k}\right)^{2}$, fulfills

$\frac{\partial I}{\partial t}-V_{\mathrm{A}} \frac{\partial I}{\partial x}=\sigma I$

The wave-frame intensity $\tilde{I}(\tilde{k}, x)$ is obtained from the Lorentz transformation of the electromagnetic fields and wavenumber noting that in the wave frame both the wave frequency and the wave-electric field vanish. Thus,

$\tilde{k}=k / \Gamma_{\mathrm{A}} ; \quad \Gamma_{\mathrm{A}}=\sqrt{1+v_{\mathrm{A}}^{2} / c^{2}}$

$\tilde{I}(\tilde{k})=I\left(\Gamma_{\mathrm{A}} \tilde{k}\right) / \Gamma_{\mathrm{A}}$.

The wave-frame intensity is, therefore, governed by the same equation as the fixed-frame intensity. In the steady state, this gives Eq. (7) for the wave-frame intensity.

The growth rate for a non-relativistic $V_{\mathrm{A}}$ can be found in the literature (e.g., Skilling 1975). We derive it here for a general $V_{\mathrm{A}}<c$ from the particle motion. As the particle scatters off the Alfvén wave by an amount of $\Delta \tilde{\mu}$, its total energy $E$ in the plasma frame is changed by $\Delta E=-\Gamma_{\mathrm{A}} V_{\mathrm{A}} \tilde{p} \Delta \tilde{\mu}$. Particles, hence, lose energy in the plasma frame at the rate

$-\frac{\langle\Delta E\rangle}{\Delta t}=\Gamma_{\mathrm{A}} V_{\mathrm{A}} \tilde{p} \frac{\langle\Delta \tilde{\mu}\rangle}{\Delta t}=\Gamma_{\mathrm{A}} V_{\mathrm{A}} \tilde{p} \frac{\tilde{E}}{E} \frac{\partial \tilde{D}_{\mu \mu}^{(j)}}{\partial \tilde{\mu}}$.

Thus, the blob-frame energy density of the particles decreases at the rate

$$
\begin{aligned}
-\frac{\mathrm{d} U_{j}}{\mathrm{~d} t} & =\int \mathrm{d}^{3} p \Gamma_{\mathrm{A}} V_{\mathrm{A}} \tilde{p} \frac{\tilde{E}}{E} \frac{\partial \tilde{D}_{\mu \mu}^{(j)}}{\partial \tilde{\mu}} f_{j} \\
& =\int \mathrm{d}^{3} \tilde{p} \Gamma_{\mathrm{A}} V_{\mathrm{A}} \tilde{p} \frac{\partial \tilde{D}_{\mu \mu}^{(j)}}{\partial \tilde{\mu}} f_{j} \\
& =-\int \mathrm{d}^{3} \tilde{p} \Gamma_{\mathrm{A}} V_{\mathrm{A}} \tilde{p} \tilde{D}_{\mu \mu}^{(j)} \frac{\partial f_{j}}{\partial \tilde{\mu}}
\end{aligned}
$$

The decrease of the particle-energy density must lead to an equal increase of the wave-energy density, $\int \mathrm{d} k I / 4 \pi$, i.e.,

$\frac{1}{4 \pi} \int \mathrm{d} k \sigma I=-\sum_{j=e, p} \int \mathrm{d}^{3} \tilde{p} \Gamma_{\mathrm{A}} V_{\mathrm{A}} \tilde{p} \tilde{D}_{\mu \mu}^{(j)} \frac{\partial f_{j}}{\partial \tilde{\mu}}$,

which we evaluate for the distribution given in Eq. (1) as

$\frac{1}{4 \pi} \int \mathrm{d} k \sigma I=-\sum_{j=e, p} \int \mathrm{d} \tilde{\mu} \Gamma_{\mathrm{A}} V_{\mathrm{A}} \tilde{\Gamma} m_{j} \tilde{V} \tilde{D}_{\mu \mu}^{(j)} \frac{\partial F_{j}}{\partial \tilde{\mu}}$.
Plugging in the scattering rate from Eq. (4), we get

$$
\begin{aligned}
& \frac{\Gamma_{\mathrm{A}}}{4 \pi} \int \mathrm{d} \tilde{k} \sigma \tilde{I}=-\sum_{j=e, p} \int \mathrm{d} \tilde{\mu} V_{\mathrm{A}} \tilde{\Gamma} m_{j} \tilde{V} \\
& \quad \times \frac{\pi \Omega_{j}^{2}}{2 B^{2} \tilde{\Gamma}^{2}}\left(1-\tilde{\mu}^{2}\right) \int \mathrm{d} \tilde{k} \tilde{I}(\tilde{k}, x) \delta\left(\tilde{k} \tilde{V} \tilde{\mu}+\frac{\Omega_{j}}{\tilde{\Gamma}}\right) \frac{\partial F_{j}}{\partial \tilde{\mu}} .
\end{aligned}
$$

Changing the order of integration we get

$$
\begin{aligned}
& \int \mathrm{d} \tilde{k} \sigma \tilde{I}=\int \mathrm{d} \tilde{k}\left[-\frac{4 \pi}{\Gamma_{\mathrm{A}}} \sum_{j=e, p} \tilde{I}(\tilde{k}, x) V_{\mathrm{A}} \tilde{\Gamma} m_{j} \tilde{V}\right. \\
& \left.\times \int \mathrm{d} \tilde{\mu} \frac{\pi \Omega_{j}^{2}}{2 B^{2} \tilde{\Gamma}^{2}}\left(1-\tilde{\mu}^{2}\right) \delta\left(\tilde{k} \tilde{V} \tilde{\mu}+\frac{\Omega_{j}}{\tilde{\Gamma}}\right) \frac{\partial F_{j}}{\partial \tilde{\mu}}\right] .
\end{aligned}
$$

Thus, the growth rate is

$$
\begin{aligned}
\sigma= & -\frac{4 \pi}{\Gamma_{\mathrm{A}}} \sum_{j=e, p} V_{\mathrm{A}} \tilde{\Gamma} m_{j} \tilde{V} \\
& \times \int \mathrm{d} \tilde{\mu} \pi \frac{\Omega_{j}^{2}}{2 B^{2} \tilde{\Gamma}^{2}}\left(1-\tilde{\mu}^{2}\right) \delta\left(\tilde{k} \tilde{V} \tilde{\mu}+\frac{\Omega_{j}}{\tilde{\Gamma}}\right) \frac{\partial F_{j}}{\partial \tilde{\mu}} \\
= & -\frac{4 \pi}{\Gamma_{\mathrm{A}}} \sum_{j=e, p} V_{\mathrm{A}} \tilde{\Gamma} m_{j} \tilde{V} \frac{\left|\mu_{j}(\tilde{k})\right|}{|\tilde{k}|} H\left(|\tilde{k}|-\tilde{R}_{j}\right) \\
& \times\left(\pi \frac{\Omega_{j}^{2}}{2 B^{2} \tilde{V}|\tilde{\mu}| \tilde{\Gamma}^{2}}\left(1-\tilde{\mu}^{2}\right) \frac{\partial F_{j}}{\partial \tilde{\mu}}\right)_{\tilde{\mu}=\mu_{j}(\tilde{k})} .
\end{aligned}
$$

Multiplying both sides by $\tilde{I}(\tilde{k})=\tilde{I}\left[k_{j}(\tilde{\mu})\right]_{\tilde{\mu}=\mu_{j}(\tilde{k})}$ results in Eq. (7). Replacing the mono-energetic distribution by a spectrum is straight-forward and left as an exercise to the interested reader.

In case of large-angle scattering, the wave-growth rate is evaluated very similarly. Since the scattering events now totally isotropize the particles, we have $\langle\Delta \tilde{\mu}\rangle=-\tilde{\mu}$ giving $-\langle\Delta E\rangle=$ $-\Gamma_{\mathrm{A}} V_{\mathrm{A}} \tilde{p} \tilde{\mu}$ per scattering. The rate of scatterings in the wave frame is $1 / \tau$ giving $\tilde{E} /(E \tau)$ in the blob frame. In addition to the scattered particles described by $F$ in our model, we have to include the beamed source particles. Thus,

$-V_{\mathrm{A}} \frac{\partial U}{\partial x}=-\int \mathrm{d}^{3} p \Gamma_{\mathrm{A}} V_{\mathrm{A}} \tilde{p} \tilde{\mu} \frac{f}{\tau} \frac{\tilde{E}}{E}$,

which is easiest to evaluate in the wave frame, where

$f=\frac{\delta\left(\tilde{p}-\tilde{\Gamma} m_{j} \tilde{V}\right)}{2 \pi \tilde{p}^{2}}[F(x, \tilde{\mu})+\delta(\tilde{\mu}+1) Q(x) \tau(x)]$

We get

$$
\begin{aligned}
-V_{\mathrm{A}} \frac{\partial U}{\partial x} & =-\Gamma_{\mathrm{A}} V_{\mathrm{A}} \tilde{\Gamma} m_{j} \tilde{V} \int_{-1}^{+1} \mathrm{~d} \tilde{\mu} \tilde{\mu} \frac{F+\delta(\tilde{\mu}+1) Q \tau}{\tau} \\
& =\Gamma_{\mathrm{A}} V_{\mathrm{A}} \tilde{\Gamma} m_{j}\left(\tilde{V} Q-\frac{S}{\tau}\right)
\end{aligned}
$$

i.e., Eq. (37). 
R. Vainio et al.: Particle transport effects in relativistic collision fronts, Online Material p 3

\section{Appendix B: Consistency of the quasi-linear modeling}

In this appendix, we discuss the consistency of the quasilinear modeling with the assumption of particle isotropization at $x \rightarrow-\infty$ making various assumptions on the transmission coefficient and the shape of the distribution function at $x=0$.

Let us start with the case of $T_{j}=4 \tilde{V} V_{\mathrm{A}} /\left(\tilde{V}+V_{\mathrm{A}}\right)^{2}$. If the backscattered part of the particle distribution at $x=0$ would be isotropic - a simplifying, albeit somewhat unphysical assumption - it would read

$$
\begin{gathered}
F_{j}\left(0, \tilde{\mu}>V_{\mathrm{A}} / \tilde{V}\right)=\left(1-T_{j}\right) \tilde{n}_{\mathrm{i}} \frac{2 \tilde{V}\left(\tilde{V}+V_{\mathrm{A}}\right)}{\left(\tilde{V}-V_{\mathrm{A}}\right)^{2}} \\
T_{j}=4 \tilde{V} V_{\mathrm{A}} /\left(\tilde{V}+V_{\mathrm{A}}\right)^{2} \\
\stackrel{n_{\mathrm{i}}}{=} \frac{2 \tilde{V}}{\tilde{V}+V_{\mathrm{A}}}=F_{j}(-\infty) .
\end{gathered}
$$

In this case, no waves would be generated by particles at $\tilde{\mu}>$ $V_{\mathrm{A}} / \tilde{V}$. Note that if the particle distribution is isotropic in the backward-wave frame, it still damps the forward waves so in this sense the result is consistent with the assumption of there being only backward waves in the system.

In case of an isotropic boundary distribution at $\tilde{\mu}>V_{\mathrm{A}} / \tilde{V}$, and for a general value of $T_{j}$ the spectrum of self-generated waves at wavenumbers resonant with $\tilde{\mu}>V_{\mathrm{A}} / \tilde{V}$ is given by

$$
\begin{gathered}
\tilde{I}(\tilde{k},-\infty)-\tilde{I}_{0}(\tilde{k})=4 \pi\left(\tilde{V}+V_{\mathrm{A}}\right) \tilde{n}_{\mathrm{i}} \sum_{j=e, p} H\left(|\tilde{k}|-\tilde{R}_{j}^{-1}\right) \\
\times \frac{m_{j}\left|\Omega_{j}\right|}{\tilde{k}^{2}}\left[1-T_{j} \frac{\left(\tilde{V}+V_{\mathrm{A}}\right)^{2}}{4 \tilde{V} V_{\mathrm{A}}}\right]\left[1-\frac{\left(\tilde{V} \mu_{j}-V_{\mathrm{A}}\right)^{2}}{\left(\tilde{V}-V_{\mathrm{A}}\right)^{2}}\right] .
\end{gathered}
$$

We have plotted the value of $\tilde{k}^{2}\left[\tilde{I}(\tilde{k},-\infty)-\tilde{I}_{0}(\tilde{k})\right]$ due to each particle species in Fig. B.1 as a function of resonant $\tilde{\mu}=\mu_{j}$ for a few values of $T_{j} \equiv \alpha_{1} 4 V_{\mathrm{A}} \tilde{V} /\left(\tilde{V}+V_{\mathrm{A}}\right)^{2}$. The wave spectrum for the isotropic boundary distribution is negative at $\mu_{j}$ greater than the value plotted in Fig. 2 in all cases, but a function with a positive slope, $\partial F(0, \mu) / \partial \mu>0$, could produce a positive part to the spectrum also at positive pitch-angle cosines. This is easily seen by considering the extreme (and unphysical) case of $F\left(\tilde{\mu}>V_{\mathrm{A}} / \tilde{V}\right) \propto \delta(\tilde{\mu}-1)$, which yields the spectrum given by Eq. (14) also for $V_{\mathrm{A}} / \tilde{V}<\tilde{\mu}<1$.

Scattering by self-generated waves is not the only way particles can cross the regions near $\tilde{\mu}=0$ and $\tilde{\mu}=V_{\mathrm{A}} / \tilde{V}$. Within the quasi-linear scattering model, if $\tilde{V} / V_{\mathrm{A}}>m_{\mathrm{p}} / m_{\mathrm{e}}=1836$, protons near $\tilde{\mu}=V_{\mathrm{A}} / \tilde{V}$ can also be in resonance with waves generated by electrons, so the condition for positive total wave intensity is more relaxed. This is also true for a finite intensity of the background waves, which should have a spectral index $q<2$ for efficient scattering near $\tilde{\mu}=0$.

In the present model, for $\tilde{V} / V_{\mathrm{A}}<m_{\mathrm{p}} / m_{\mathrm{e}}$, the upper limit for the proton transmission coefficient is determined by the background wave spectrum. Now the self-generated part of the wave spectrum can also be negative; if the background spectrum has the same $\propto \tilde{k}^{-2}$ dependence as the asymptotic self-generated

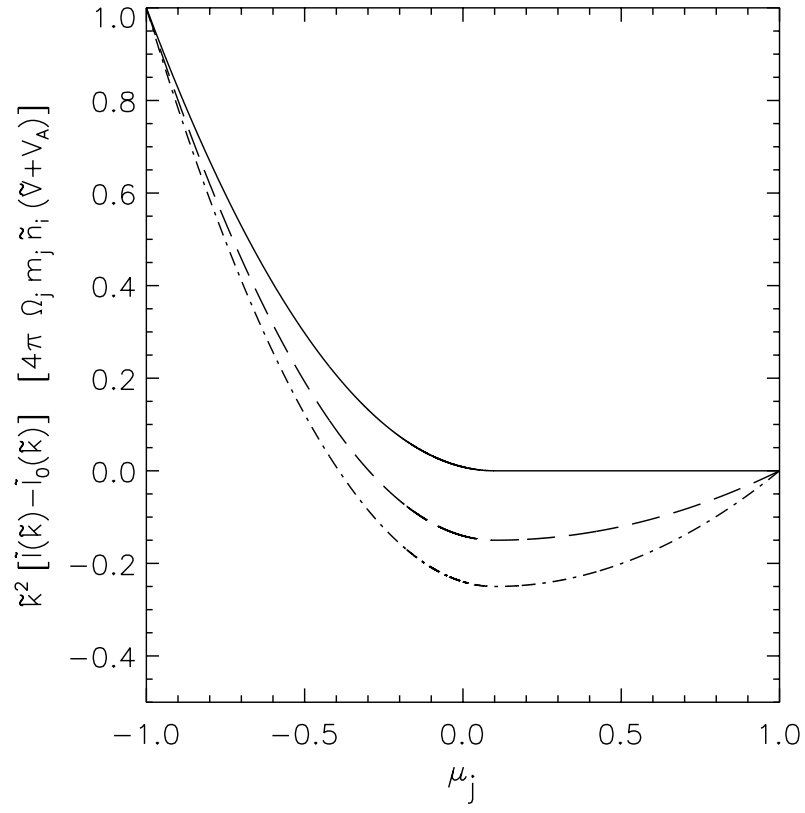

Fig. B.1. Wave spectrum generated by protons (or electrons) as a function of resonant pitch-angle cosine for $V_{\mathrm{A}} / \tilde{V}=0.1$ and $T_{j}=$ $\alpha_{1} 4 \tilde{V} V_{\mathrm{A}} /\left(\tilde{V}+V_{\mathrm{A}}\right)^{2}$ with $\alpha_{1}=1.0$ (solid curve), 1.15 (dashed curve), and 1.25 (dot-dashed curve). Note that the area under each curve is proportional to the wave-energy density.

spectrum, for example, the condition for positive total spectrum near $\tilde{k}=-\Omega_{\mathrm{p}} / \tilde{\Gamma} V_{\mathrm{A}}$ is

$T_{j}<\left(1+r_{I}\right) \frac{4 \tilde{V} V_{\mathrm{A}}}{\left(\tilde{V}+V_{\mathrm{A}}\right)^{2}}$,

where

$r_{I}=\frac{\tilde{k}^{2} I_{0}(\tilde{k})}{4 \pi m_{\mathrm{p}}\left|\Omega_{\mathrm{p}}\right|\left(\tilde{V}+V_{\mathrm{A}}\right) \tilde{n}_{\mathrm{i}}}$

measures the ratio of the background wave spectrum to selfgenerated one. Evaluating the integrated resonant background wave intensity,

$$
\begin{aligned}
\left(\delta \tilde{B}_{0}\right)^{2} & =2 \int_{\tilde{R}_{\mathrm{p}}^{-1}}^{\infty} \mathrm{d} \tilde{k} \tilde{I}_{0}(\tilde{k})=2 r_{I} 4 \pi m_{\mathrm{p}} \tilde{\Gamma} \tilde{V}\left(\tilde{V}+V_{\mathrm{A}}\right) \tilde{n}_{\mathrm{i}} \\
& \approx 2 r_{I} B^{2} \Gamma^{2} \frac{n_{\mathrm{i}}^{*}}{n_{b}} \frac{V^{2}}{V_{\mathrm{A}}^{2}}
\end{aligned}
$$

where the last equation holds for a non-relativistic $V_{\mathrm{A}}$. For typical parameters, $V / V_{\mathrm{A}}=10^{2}=\Gamma$ and $n_{\mathrm{i}}^{*} / n_{b}=10^{-8}$, we obtain

$r_{I} \approx \frac{\left(\delta B_{0}\right)^{2}}{2 B^{2}} \ll 1$,

which means that the correction to the maximum transmission coefficient is small for a steep $\left(\propto \tilde{k}^{-2}\right)$ background wave spectrum. We note, therefore, that if the background spectrum is not substantially harder than the self-generated one, the time scale associated with crossing $\tilde{\mu}=0$ is much larger than the time scale related to the isotropization of the particles inside one hemisphere. Note also that the ratio of these time scales depends on the applied turbulence model: for example, relativistic 
particles scattering off transverse cold-plasma waves resonate with finite wavenumbers everywhere (Vainio 2000). The dispersive effects, therefore, lead to a finite amount of scattering across the whole phase space.

Even if the resonance gap at $\tilde{\mu}=0$ is filled, there is still the possibility of inefficient crossing of $\tilde{\mu}=V_{\mathrm{A}} / \tilde{V}$. Arguing that particles become isotropic at $\tilde{\mu}<V_{\mathrm{A}} / \tilde{V}$ or $\tilde{\mu}>V_{\mathrm{A}} / \tilde{V}$ faster than crossing this boundary now yields exactly the same transmission coefficient as for an isotropic particle distribution. In this case, particle distribution at the boundary would, indeed, be isotropic at $\tilde{\mu}>V_{\mathrm{A}} / \tilde{V}$ and there would be no self-generated waves resonant with these particles, which may contradict the above assumption of fast scattering at $\tilde{\mu}>V_{\mathrm{A}} / \tilde{V}$. This alternative, therefore, does not seem self-consistent. 